\title{
SYNTHESIZED SPECTRA OF OPTICALLY THIN EMISSION LINES PRODUCED BY THE BIFROST STELLAR ATMOSPHERE CODE, INCLUDING NONEQUILIBRIUM IONIZATION EFFECTS: A STUDY OF THE INTENSITY, NONTHERMAL LINE WIDTHS, AND DOPPLER SHIFTS
}

\author{
K. Olluri ${ }^{1}$, B. V. Gudiksen ${ }^{1}$, V. H. Hansteen ${ }^{1}$, and B. De Pontieu ${ }^{1,2}$ \\ ${ }^{1}$ Institute of Theoretical Astrophysics, University of Oslo, P. O. Box 1029 Blindern, NO-0315 Oslo, Norway; kosovare.olluri@astro.uio.no \\ ${ }^{2}$ Lockheed Martin Solar and Astrophysics Lab, Org. A021S, Bldg. 252, 3251 Hanover Street, Palo Alto, CA 94304, USA \\ Received 2013 November 27; accepted 2014 December 25; published 2015 March 13
}

\begin{abstract}
In recent years realistic 3D numerical models of the solar atmosphere have become available. The models attempt to recreate the solar atmosphere and mimic observations in the best way, in order to make it possible to couple complicated observations with physical properties such as the temperatures, densities, velocities, and magnetic fields. We here present a study of synthetic spectra created using the Bifrost code in order to assess how well they fit with previously taken solar data. A study of the synthetic intensity, nonthermal line widths, Doppler shifts, and correlations between any two of these three components of the spectra first assuming statistical equilibrium is made, followed by a report on some of the effects nonequilibrium ionization will have on the synthesized spectra. We find that the synthetic intensities compare well with the observations. The synthetic observations depend on the assumed resolution and point-spread function (PSF) of the instrument, and we find a large effect on the results, especially for intensity and nonthermal line width. The Doppler shifts produce the reported persistent redshifts for the transition region (TR) lines and blueshifts for the upper TR and corona lines. The nonthermal line widths reproduce the well-known turnoff point around $(2-3) \times 10^{5} \mathrm{~K}$, but with much lower values than those observed. The nonthermal line widths tend to increase with decreasing assumed instrumental resolution, also when nonequilibrium ionization is included. Correlations between the nonthermal line width of any two TR line studies as reported by Chae et al. are reproduced, while the correlations of intensity to line width are reproduced only after applying a PSF to the data. Doppler shift correlations reported by Doschek for the TR lines and correlations of Doppler shift to nonthermal line width of the Fe $\mathrm{XII}_{19.5}$ line reported by Doschek et al. are reproduced.
\end{abstract}

Key words: line: profiles - magnetohydrodynamics (MHD) - methods: numerical - methods: statistical - Sun: transition region - Sun: UV radiation - techniques: spectroscopic

Supporting material: animation

\section{INTRODUCTION}

The outer layers of the Sun have proven difficult to understand despite more than 50 years of steadily improving observations. This is especially true when considering the upper chromosphere, the transition region (TR), and the lower corona. This region contains a number of complex physical transitions that have impeded detailed insight: radiative emission goes from being optically thick to being optically thin. The plasma $\beta$ parameter drops rapidly with height, as the plasma goes from gas pressure dominated to magnetic pressure dominated. Hydrogen becomes fully ionized, while the timescale of hydrogen ionization becomes longer than typical dynamic timescales. In the TR, the temperature rises from $10^{4}$ to $10^{6} \mathrm{~K}$ over very short distances, and even small velocities can cause nonequilibrium ionization to play an important role for the ions that dominate radiative losses at temperatures where hydrogen is fully ionized. With all this in mind, it has been difficult to isolate possible culprits when reasonablelooking models have failed to reproduce even the basic observational constraints (Mariska 1992; Wilhelm et al. 2007).

The Interface Region Imaging Spectrograph (IRIS; De Pontieu et al. 2014), launched on 2013 June 28, gives highquality observations of the lower chromosphere and lower TR. The probe observes TR lines with the highest cadence, spatial resolution, and spectral resolution so far-at $1 \mathrm{~s}, \frac{1}{3}$, and $28 \times 10^{-4} \mathrm{~nm}$, respectively. This is a significant improvement, and the possibility of making progress in understanding the upper chromosphere and lower TR is in sight.

Along with IRIS observations, and as part of the IRIS data product, state-of-the-art 3D numerical models of the solar atmosphere produced with the Bifrost code (Gudiksen et al. 2011) are publicly available. This data product includes time snaps of the MHD variables $(\rho, \mathbf{u}, e, \mathbf{B})$ and assorted associated variables (temperature, pressure, etc). In addition, synthetic line profiles calculated from these snapshots of the most important spectral lines observable by IRIS are provided. The models are to be used in parallel with the observations to achieve the mission goals of understanding the energy and mass transfer of this "interface" region. However, before this work is initiated, we need to ensure that the simulated spectra (and therefore the models from which they are derived) are close enough to already-obtained observations to provide a meaningful basis of comparison. In this paper we will critically go through the synthesized lines observable by IRIS, as well as selected lines observed by the high-resolution telescope spectrometer (HRTS), Solar and Heliospheric Observatory $(\mathrm{SOHO}) / \mathrm{SUMER}$, and Hinode/EIS, and discuss how well these observations are mirrored by the synthetic observables.

The upper chromosphere and TR radiate mainly in the ultraviolet and extreme-ultraviolet, and observations necessarily require balloon- or space-borne instrumentation. Among the many instruments used to study this region, we find UV spectrometer and polarimeter (UVSP) on Solar Maximum Mission (SMM; Woodgate et al. 1980), the HRTS instrument 
(Bartoe et al. 1986; Brueckner et al. 1986), SOHO/SUMER (Wilhelm et al. 1995) and SOHO/CDS (Harrison et al. 1995), TRACE (Handy et al. 1999), and Hinode/EIS (Culhane et al. 2007). The observational facts of the pre-SOHO era are well summarized by Mariska (1992), while Wilhelm et al. (2007) have collected and summarized the findings of the two decades that have passed since then.

The large number of observations made with these instruments include a variety of solar features with optically thin lines covering formation temperatures from the upper chromosphere, TR, and corona. Despite the tremendous amount of data available, the interface region is still not understood, in the sense that most models of the region do not reproduce even the average lower moments of the observations, i.e., the intensity, Doppler shift, and line width. That implies that the basic idea underlying these models, that TR emission largely comes from structures connected via the magnetic field to the hot corona above and where the temperature profile is determined from the properties of thermal conduction along the magnetic field, is incorrect, or must be modified in some way.

Studies of spectral synthesis from 1D models are plentiful (Klimchuk et al. 1997; Walsh \& Galsgaard 2000; Spadaro et al. 2003; Warren 2006; Reale 2010; Winebarger et al. 2011, to name but a few), and many (Warren 2006; Klimchuk et al. 2012; Chitta et al. 2013) have compared the models with the observed differential emission measure (DEM), which essentially gives the amount of emitting material in the atmosphere as a function of temperature (Pottasch 1964). The key features of the observed solar DEM are a negative slope at low temperatures, with a minimum near $2 \times 10^{5} \mathrm{~K}$, followed by a maximum near $10^{6} \mathrm{~K}$, after which one again finds a negative slope toward higher temperatures. The negative slope at low TR temperatures implies that there is much more material emitting at these temperatures than 1D models readily can reproduce.

Also, average observed Doppler shifts have been difficult to understand; they show a persistent redshift for lines formed at TR temperatures (Doschek et al. 1976), with a maximum average redshift of some $10 \mathrm{~km} \mathrm{~s}^{-1}$ found for lines formed at $10^{5} \mathrm{~K}$. At higher temperatures the redshifts decrease, with indications of average blueshifts for coronal lines (Peter et al. 1999). Explanations for this finding based on the idea of cooling, returning, spicular material have abounded (Mariska et al. 1978; Athay 1984; Hansteen \& Wikstøl 1994; Wilhelm 2000; Doyle et al. 2002; Bradshaw \& Cargill 2010), but it has proven difficult to reproduce the observational values with any 1D hydrodynamic model. An alternate 1D loop model of coronal nanoflare-generated shock waves (Hansteen 1993) does produce net average redshifts of the TR lines, but with a significantly smaller amplitude than what is observed.

The spectral lines formed in the middle TR show large unresolved velocities resulting in large line widths, varying from $10 \mathrm{~km} \mathrm{~s}^{-1}$ in the lower TR $\left(3 \times 10^{4} \mathrm{~K}\right)$ to $30 \mathrm{~km} \mathrm{~s}^{-1}$ in the middle TR $\left(2 \times 10^{5} \mathrm{~K}\right)$ and then decreasing at higher temperatures (Chae et al. 1998, and references therein).

Doschek (2006) found strong positive correlations between the Doppler shifts of spectral lines formed at similar temperatures low in the TR, but that the correlation weakens as the difference in formation temperature increases and completely disappears between low TR lines and coronal lines. Hara et al. (2008) also found strong positive correlations between Doppler shifts and nonthermal line widths at disk center, while Chae et al. (1998) found strong positive correlations between the nonthermal line widths of lines formed below $2 \times 10^{5} \mathrm{~K}$, as well as positive correlations between the intensity and the nonthermal line widths for the same lines, supporting previous studies by Dere \& Mason (1993). Akiyama et al. (2003), on the other hand, found no correlations between the intensity and the nonthermal line widths, supporting previous studies by Athay et al. (1983).

While it has proven difficult to reconcile 1D models with the observations, 3D solar atmosphere models seem to do a better job. Peter et al. (2006) were the first to reproduce the overall shape of the DEM from their 3D MHD model, but they failed to reproduce the high- and low-temperature ends of the curve. Peter et al. (2006) and later, at higher resolution, Hansteen et al. (2010) found that their 3D models reproduce the average redshifts at lower TR temperatures, also finding blueshifts in hotter coronal emission. In a follow-up paper Guerreiro et al. (2013) explain this as a result of the preponderance of lowlying cool loops - a dynamic version of Antiochos's (1982) idea of low-lying loops dominating lower TR emission.

Only Peter et al. (2006) have had significant analysis of the nonthermal line widths derived from synthesized spectra. But their results poorly match the observed values in the corona and lower TR, and they are not able to reproduce the reported peak at $2 \times 10^{5} \mathrm{~K}$. In general, models give much smaller line widths than what is observed.

Finally, we note that line intensities and profiles show a strong time variability, with short timescales especially prevalent in the middle TR (Hansteen et al. 2000; Brković et al. 2003). When the timescales of the dynamics are shorter than the typical ionization and recombination times of the emitting ions, nonequilibrium ionization becomes important (Olluri et al. 2013b). This makes the exposure time used by the different instruments a factor in the results. A Bifrost module that can be used to compute the ionization state of the most important radiating ions in the TR has already been used to analyze the behavior of IRIS relevant density-sensitive line ratios (Olluri et al. 2013a).

In this paper, synthetic spectra are computed of the IRIS C II

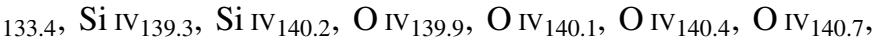
and $\mathrm{Fe} \mathrm{XII}_{134.9}$ lines, the EIS EUV He $\mathrm{II}_{25.6}$ and Fe $\mathrm{XII}_{19.5}$ lines, and the $\mathrm{O} \mathrm{VI}_{103.1}$ line connecting the middle $\mathrm{TR}$ to the corona, under optically thin conditions. We investigate the line intensity, the line width, and the line shift and see how well they reproduce observed data from HRTS, SUMER, and EIS. In addition to studying the results averaged over the entire simulated model, we take a closer look at the synthetic observables over regions of high magnetic field concentration, i.e., a loop footpoint, and over magnetic loop apexes, investigating the influence of the magnetic field topology on the spectra.

We begin with a description of the numerical model computed with Bifrost in Section 2 and the method of synthesizing the UV spectra in Section 3. In Section 4 we discuss the spectral line selection for the study. In Sections 5-7 we discuss the results of synthesized line intensity, nonthermal line widths, and Doppler velocities, respectively. In Section 8 we investigate various correlations in the observables and how they compare to similar results from actual observations. Finally, in Section 9 we discuss how including nonequilibrium ionization affects the observables, ending the study with a discussion of the results in Section 10. 

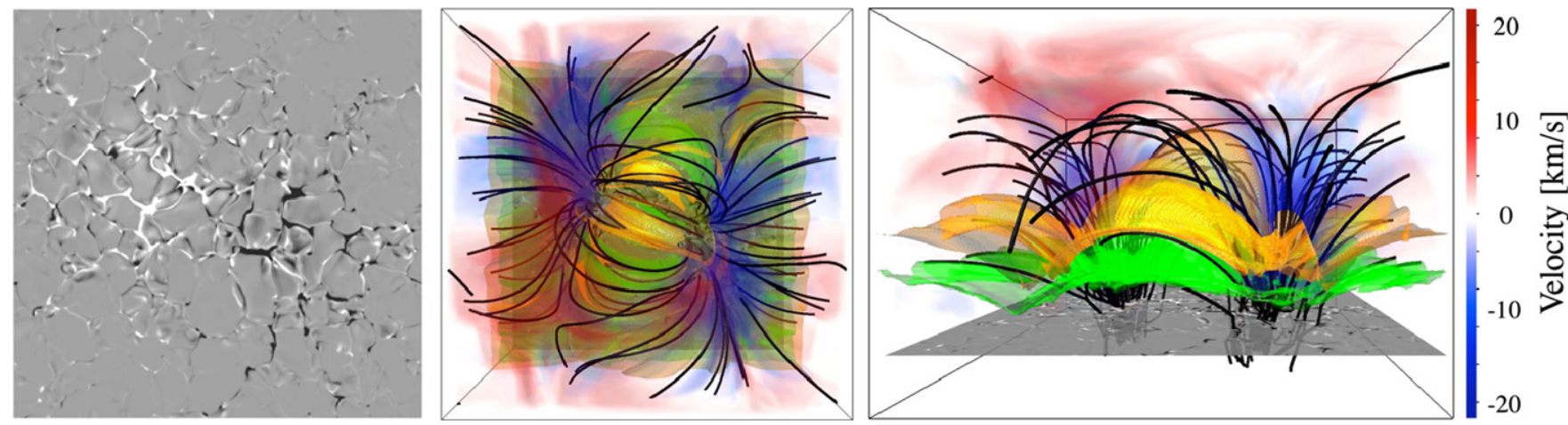

Figure 1. Snapshot of the simulation, 23 minutes after $t=0 \mathrm{~s}$. In an " $x z$ " view in the right panel and in an " $x y$ " view in the middle panel. The left panel displays the vertical magnetic field at photosphere $(z=0)$ representing a "magnetogram." The blue-red color distribution in the box represents the vertical velocity. The green isosurface shows the temperature at $\log T=5$, and the yellow isosurface shows the temperature at $\log T=6$. The magnetic topology is indicated by the magnetic field lines shown as back loops, and the grayscale slice represents the strength of the magnetic field in the photosphere, where there are two opposite field concentrations along the diagonal of the box.

\section{THE NUMERICAL MODEL}

The investigations of synthetic spectra in this paper are based on 3D MHD models created with the stellar atmosphere code Bifrost (Gudiksen et al. 2011), including the nonequilibrium ionization solver described in Olluri et al. (2013b).

Bifrost solves the full radiative MHD equations on a staggered grid. The radiative flux divergence in the photosphere and chromosphere is obtained using the method of opacity binning developed by Nordlund (1982), and 3D scattering is solved by methods developed by Skartlien (2000). The radiative losses in the photosphere drive convection that is the ultimate source of the Poynting flux that heats the outer atmospheric layers of the modeled atmosphere. The effective temperature of the model is close to solar, $T_{\text {eff }}=5780 \mathrm{~K}$. In the upper chromosphere and lower TR nonLTE radiative losses are modeled using the recipes derived by Carlsson \& Leenaarts (2012), while in the TR and corona optically thin radiative cooling is assumed. Thermal conduction along magnetic field lines is included using an implicit operator as described by Hansteen \& Gudiksen (2005) and Gudiksen et al. (2011).

The synthetic atmosphere models under consideration span the atmosphere from the upper layers of the convection zone, $2.4 \mathrm{Mm}$ below the photosphere, to $14.4 \mathrm{Mm}$ above, representing the lower corona. The particular experiment used for this study models the solar atmosphere on a computational grid of $512 \times 512 \times 496$ points, spanning $24 \times 24 \times 17 \mathrm{Mm}^{3}$, with a uniform horizontal grid spacing of $47.6 \mathrm{~km}$ in the $x$ and $y$ directions and a nonuniform grid spacing in the vertical $z$ direction. The nonuniform grid spacing is set up to resolve the strong gradients in the photosphere and chromosphere, ranging from a minimum of $18 \mathrm{~km}$ in the photosphere to the TR, increasing to $80 \mathrm{~km}$ in the corona and convection zone. The "high-resolution" $18 \mathrm{~km}$ zone extends from $800 \mathrm{~km}$ below the photosphere to $5 \mathrm{Mm}$ above the photosphere, in practice covering almost all of the high temperature gradient TR plasma. Model boundaries are periodic in the horizontal directions. The lower convection zone boundary allows outflowing material to leave the computational box, while the entropy of inflowing material is set so as to maintain an effective temperature that is close to solar. At the upper coronal boundary, (nearly) transparent characteristic boundary conditions are applied, in principle allowing waves to propagate through without reflection, while at the same time the temperature gradient is forced to zero.

The model described in this paper has essentially the same magnetic field strength and topology as the model released as part of the IRIS data set (M. Carlsson, in preparation), but with the difference that in the model described here hydrogen ionization is assumed to be in LTE. A more detailed description of the model can therefore be found in the IRIS technical note $33^{3}$ and references therein.

\subsection{This Simulation}

In order to construct the simulations described here, a fully convective hydrodynamic model is seeded with a potential magnetic field. After being subjected to convective and photospheric motions for some 20 minutes, the magnetic field reaches a quasi-steady stressed state, and in the low- $\beta$ upper atmosphere dissipation of magnetic energy becomes large enough that coronal temperatures are maintained in this upper part of the computational domain. The initial model $(t=0 \mathrm{~s})$ shown here was run for more than 1 hour solar time, after enough time has passed for the system to lose memory of the initial seeding of the magnetic field. We find an average unsigned flux of $48 \mathrm{G}$ in the photosphere $(z=0 \mathrm{Mm})$. The magnetic field is concentrated at two locations in the photosphere of opposite polarity, placed diagonally in the computational box, which can be seen in the three-dimensional snapshot of the model in Figure 1. The figure shows a "magnetogram" of the model in the left panel and a view of the outer atmosphere structure as seen from above and from the side in the middle and right panels, respectively.

Coronal heating in the model proceeds by a large number of small dissipation events spread throughout the magnetized chromosphere and corona. Note that the model covers many scale heights and therefore regions with very disparate timescales: from several hours near the bottom boundary to tens of minutes or less in the corona. The various layers are deviously coupled, and the quasi-steady heating rate can and will change in response to changes in the convection zone, photosphere, and chromosphere in a complicated manner. In particular, in the model described here we find that coronal heating goes from a very low level, perhaps similar to that

\footnotetext{
3 https://lmsal.com/iris_science/doc?cmd=dcur\&proj_num=IS0199\&file_type $=\mathrm{pdf}$
} 

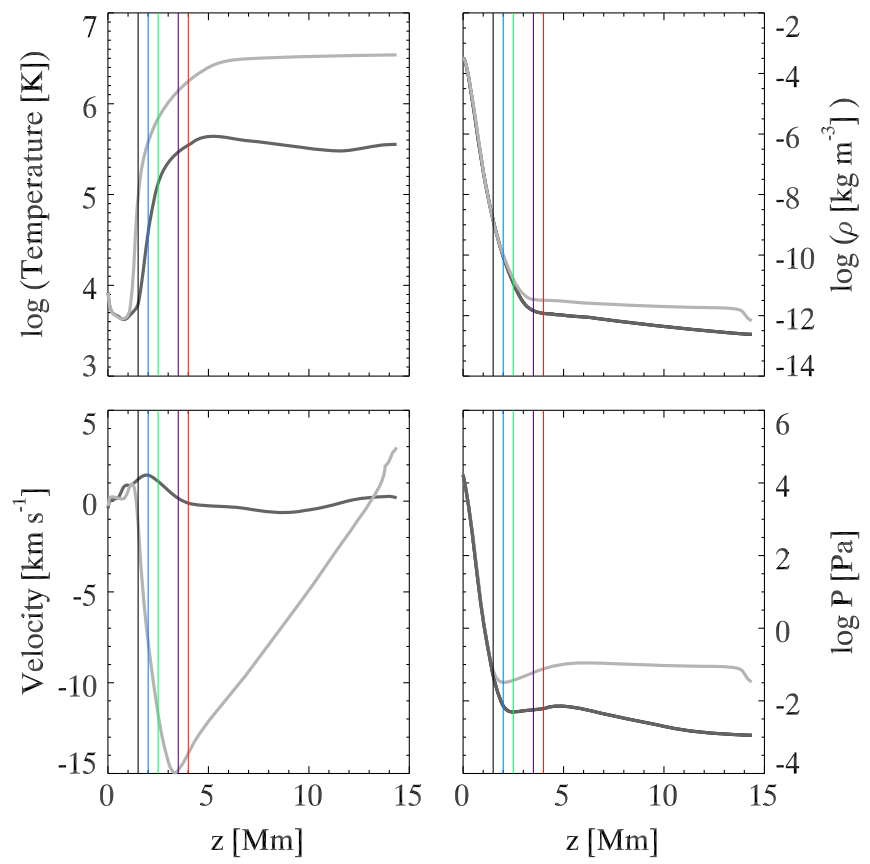

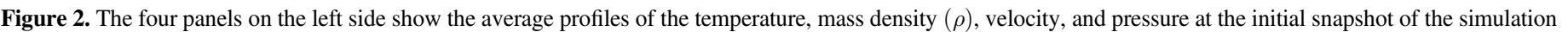

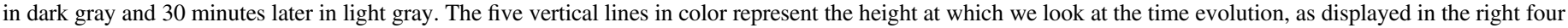
panels. Positive velocity here represents flow speeds away from the observer.

found in the very quiet Sun (QS), to a higher level closer to what could be found in regions of enhanced network, some 20 minutes after $t=0 \mathrm{~s}$. Hence, initially a low average peak temperature is found, surpassing $10^{6} \mathrm{~K}$ only in a few small regions on loops spanning the two magnetic field concentrations. The structure of the TR is shown by the green isosurface at $10^{5} \mathrm{~K}$, found at a height of some $2 \mathrm{Mm}$, though lower near the loop footpoints, and raised to some $4 \mathrm{Mm}$ in the regions between. The velocity is shown in the red-blue color haze, where blue represents the outflow velocities, appearing mostly above the regions of high magnetic field strength, i.e., the footpoints, where the field is more vertical.

In the four left panels of Figure 2 the atmospheric average profiles of the temperature, mass density, velocity, and pressure are shown. The initial atmosphere is quite cool, in the sense that the average temperature profile does not reach temperatures above $10^{6} \mathrm{~K}$, with a TR going from 6 to $350 \mathrm{kK}$, and "corona" at temperatures of only $350-450 \mathrm{kK}$. The mass density rapidly falls from $3 \times 10^{-4} \mathrm{~kg} \mathrm{~m}^{-3}$ in the photosphere to $2 \times 10^{-12} \mathrm{~kg} \mathrm{~m}^{-3}$ in the middle TR. The average velocity is largely zero at time $t=0 \mathrm{~s}$, but with average downflows of some $1 \mathrm{~km} \mathrm{~s}^{-1}$ in the midchromosphere. The pressure rapidly falls from the photosphere up to the TR, flattening out at coronal heights.

We have drawn five vertical lines, at 1.5, 2.0, 2.5, 3.5, and 4.0 Mm. These heights represent five layers of the TR where we examine the time evolution of the average quantities, drawn in the four panels on the right side of Figure 2.

The heating rate increases during the model run and the release of magnetic energy in the outer atmosphere of the model, and the average temperature rises significantly at all four chosen locations during the 30-minute simulation run. As the temperature rises, the TR is pushed further down toward the chromosphere. The average temperature of the highest layer goes from some $4 \times 10^{5} \mathrm{~K}$ to more than $1.5 \times 10^{6} \mathrm{~K}$ in the same time span.
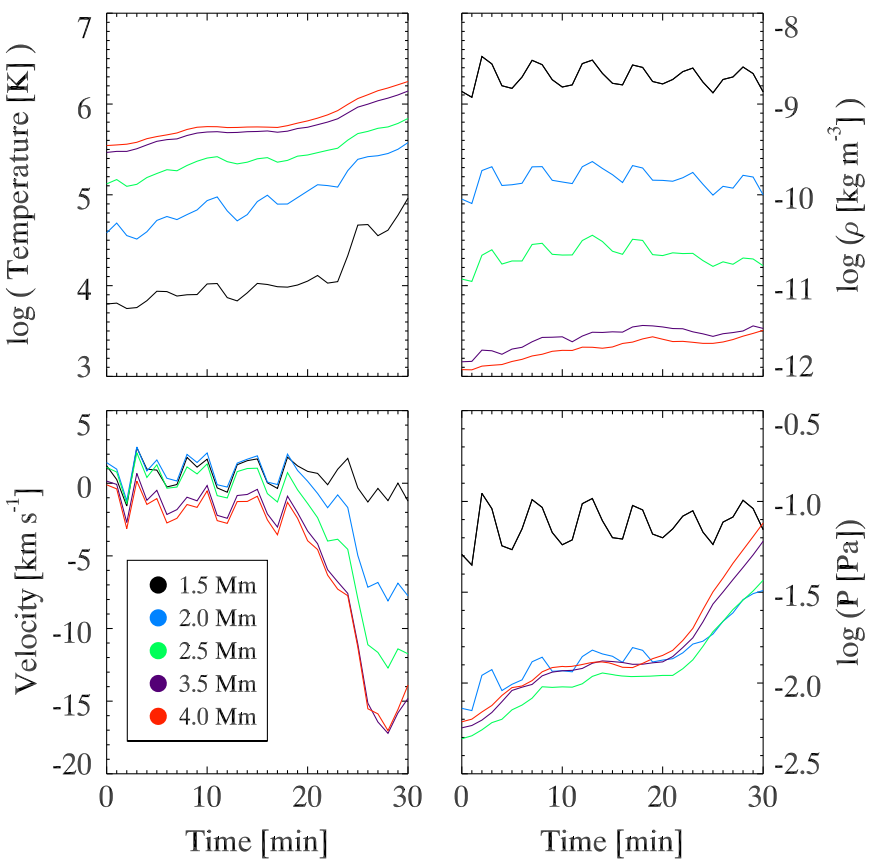

The mass density of the two lower layers does not change much, but there is a periodic variation of some 4-5 minutes with a peak-to-peak amplitude of some $0.3 \mathrm{dex}$. This variation is in part mirrored in the average temperatures, pressures, and velocities. The mass density in the two upper layers rises during the simulation. In the two top layers, the density increases by $0.5 \mathrm{dex}$ from $\log \rho \approx 10^{-12} \mathrm{~kg} \mathrm{~m}^{-3}$ to $\log \rho \approx$ $10^{-11.5} \mathrm{~kg} \mathrm{~m}^{-3}$. The amplitude of the 5 -minute fluctuations decreases slightly with time, as is evident by considering the layer at $2.5 \mathrm{Mm}$.

The average velocity fluctuates similarly for all five layers during the first 18 minutes of the simulation. The three deepest layers show positive values, indicating downflows of varying strength, and the two top layers show negative velocities, indicating upflows. Eighteen minutes into the run, the four top layers show increasing upflow velocities, increasing in strength during the following 10 minutes. This is a result of chromospheric evaporation caused by the increased temperatures and rapid changes in the pressure, as demonstrated in the bottom right panel of Figure 2 .

The initial snapshot is cool and relatively quiet. Most of the fluctuations seen in the variables are caused by a global oscillation (sound wave) with a period of roughly 5 minutes. However, after 18 minutes the heating rate, driven by the magnetic fields' nonlinear response to photospheric and convection zone motions, rises significantly. This causes all the variables to react, the temperature, density, and pressure increase, and the TR is pushed to lower levels in the atmosphere. The coronal temperature reaches $\log T=6.5$ in the final snapshot, as displayed by the light gray curves in the four left panels of Figure 2.

\section{METHODS}

We investigate the spectral line properties from the simulation by calculating the emissivity of a set of spectral 
lines at each grid point in the simulation box. We then use the emissivities and velocities from the simulation to calculate the spectrum of the line. Spatial maps of the line intensity, line shift, and line width are then obtained by calculating the profile moments with respect to the velocity. Thus, the synthesized spectra from the simulation are directly comparable to observed solar spectral lines.

\subsection{The Emissivity}

The emissivity is the contribution to the intensity at each point along the line of sight. Since we solve the rate equations, we have the level populations of both the upper and lower level of each transition, making it possible to calculate the emissivity, $\epsilon$, in the simple form

$$
\epsilon_{\nu}=\frac{h \nu}{4 \pi} n_{u} A_{\mathrm{ul}}
$$

where $\nu$ is the frequency of the spectral line, $n_{u}$ the population density of the upper level of the transition, and $A_{\mathrm{ul}}$ the Einstein spontaneous decay rate.

When assuming statistical equilibrium (SE), Equation (1) can be expressed as

$$
\epsilon_{\nu}=G\left(T, n_{e}\right) n_{e} n_{\mathrm{H}}
$$

where $n_{e}$ is the electron density, $n_{\mathrm{H}}$ is the hydrogen density, and $G\left(T, n_{e}\right)$ is the contribution function, defined as

$$
G\left(T, n_{e}\right)=\frac{h \nu_{\mathrm{ul}}}{4 \pi} \frac{A_{\mathrm{ul}}}{n_{e}} \frac{n_{u}^{i}}{n^{i}} \frac{n^{i}}{n} A_{x}
$$

where $n_{u}^{i}$ is the population density of level $u$ in ionization degree $i, n^{i}$ is the total population density of ionization degree $i$, and $n$ is the population density of a given element of interest. The abundance of the emitting element $x$ is $A_{x}=n / n_{\mathrm{H}}$, where $n_{\mathrm{H}}$ is the hydrogen density.

For Equation (2) we use CHIANTI to compute the contribution functions $G\left(T, n_{e}\right)$. We create a lookup table of $G\left(T, n_{e}\right)$ values at varying pressure values in the range $10^{11}$ $10^{18} \mathrm{~Pa}$, in a temperature range from $\log T=4.0$ to $\log T=8.0$. We then use these tables to calculate the emissivities at each point in our computational domain by interpolation in the tables with the respective temperature and electron density value in the grid point.

In this paper we perform an analysis of the synthetic observables under SE conditions using the emissivity as defined in Equation (2), and we later investigate the effect nonequilibrium ionization has on the observables by calculating the emissivity as defined in Equation (1) when investigating the results from our NEQ solver.

\subsection{Synthetic Spectral Profiles}

We assume that the emission-line profiles have a thermal width $w_{\text {th }}$ according to the temperature $T$ at every grid point in the simulation,

$$
w_{\mathrm{th}}=\left(\frac{2 k_{B} T}{m_{i}}\right)^{1 / 2}
$$

where $k_{B}$ is Boltzmanns constant and $m_{i}$ is the atomic mass of the respective ion. In addition the line is Doppler shifted according to the line of sight velocity at the respective grid point. If we look at the simulation box from above, making the
" $x y$ " plane our field of view, the $z$-axis becomes the line of sight. Then the vertical velocity $v_{z}$ is the line of sight velocity. The line profile $I_{v}$ at each grid becomes;

$$
I_{v}=\epsilon_{v} \exp \left[-\frac{\left(v-v_{v}\right)^{2}}{w_{\mathrm{th}}^{2}}\right] .
$$

Here, we have given the line profile width in units of Doppler velocities. To obtain the total spectrum of the line, we integrate along the line of sight $z$ :

$$
I_{v}^{\text {synth }}=\int_{0}^{z} I_{v} d z
$$

By doing this, we obtain two-dimensional synthetic spectral maps with the " $x y$ " plane from the computational domain, similar to disk center spectra from solar observations. The analysis of these synthetic spectra is done in similar ways to those for observed spectra: by calculating the line intensity, position, and width of $I_{v}^{\text {synth }}$ at each grid point in the " $x y$ " plane. This is done by calculating the profile moments with respect to velocity, which give the line intensity, shift, and width

$$
\begin{gathered}
I^{\text {synth }}=\int I_{v}^{\text {synth }} d v, \\
v^{\text {synth }}=\frac{1}{I^{\text {synth }}} \int v I_{v}^{\text {synth }} d v, \\
w^{\text {synth }}=\sqrt{2} \cdot\left[\frac{1}{I^{\text {synth }}} \int v^{2} I_{v}^{\text {synth }} d v-\left(v^{\text {synth }}\right)^{2}\right]^{1 / 2} .
\end{gathered}
$$

When analyzing line widths from observations, one usually subtracts the thermal line width as given by Equation (4) at peak formation temperature $T_{F}$ of the emitting ion from the computed line width (here given by Equation (9)) to obtain the nonthermal line widths,

$$
\xi^{\text {synth }}=2 \sqrt{\ln 2} \cdot\left[\left(w^{\text {synth }}\right)^{2}-w_{\text {th }}^{2}\right]^{1 / 2}
$$

but since our model atmosphere is synthetic, there might be points that have lower line widths than the thermal line width at the peak formation temperature of the emitting ion if most temperature points are along the line of sight. We will discuss this further when we present the line width results later in the paper.

\section{LINE SELECTIONS AND OBSERVATIONS}

The main goal of the paper is to compare the line intensity, line widths, and Doppler shifts from the simulation with available data from the Sun. In this paper we confine attention to the (optically thin) TR lines and place strong emphasis on the spectral lines observed with IRIS. The optically thick spectral lines observed with IRIS, in particular the $\mathrm{Mg}$ II $\mathrm{h}$ and $\mathrm{k}$ lines and the $\mathrm{C}_{\text {II }}$ lines, are discussed elsewhere (Leenaarts et al. 2013a, 2013b; Pereira et al. 2013b; B. Rathore \& M. Carlsson, in preparation).

The spectral lines selected for this study are chosen to represent emission from the lower TR up to the lower corona, covering a temperature range from $10^{4}$ to $10^{6} \mathrm{~K}$. There are, in addition, two selection criteria for the spectral lines studied; the lines should be optically thin to make it possible to synthesize the spectra as described in Section 3 without considering radiative transport, and observational data need to be available. 
Table 1

Spectral Lines Chosen for the Study

\begin{tabular}{lccc}
\hline \hline Ion & $\lambda[\mathrm{nm}]$ & $\log \mathrm{T}_{f}$ & Data Source \\
\hline Fe XII & 19.5 & 6.2 & EIS/Hinode \\
Fe XII & 134.9 & 6.2 & HRTS, SUMER/SOHO* \\
O VI & 103.1 & 5.50 & SUMER/SOHO \\
O IV & 139.9 & 5.20 & HRTS, SUMER/SOHO* \\
O IV & 140.1 & 5.20 & HRTS, SUMER/SOHO* \\
O IV & 140.4 & 5.20 & HRTS, SUMER/SOHO* \\
O IV & 140.7 & 5.20 & HRTS, SUMER/SOHO* \\
C IV & 154.8 & 5.00 & HRTS, SUMER/SOHO \\
Si IV & 139.3 & 4.80 & HRTS, SUMER/SOHO* \\
Si IV & 140.2 & 4.80 & HRTS, SUMER/SOHO* \\
C II & 133.4 & 4.65 & HRTS, SUMER/SOHO* \\
He II & 25.6 & 4.90 & EIS/Hinode \\
\hline
\end{tabular}

Note. The table lists the emitting ion, the spectral line wavelength, the peak formation temperature in ionization equilibrium, and the instrument/source of the observed data, where the asterisk indicates the spectral lines to be observed with the IRIS.

We have chosen spectral lines previously observed by HRTS, SOHO/SUMER, and Hinode/EIS. This selection allows us to perform a direct comparison with the observed properties of the line profile. The spectral lines we have selected are listed in Table 1, which lists the emitting ion, the wavelength of the spectral line, and the peak formation temperature. The He $\mathrm{II}_{25.6}$ and the $\mathrm{C}_{\mathrm{II}_{133.4}}$ lines are most likely not optically thin but have been included in spite of this, since they often are treated as optically thin. Including them will make it possible for us to evaluate this assumption.

The observations with which we compare our synthetic spectra are from the HRTS atlas (Brekke et al. 1991) for the QS and active region (AR), the SUMER atlas (Curdt et al. 2001) containing data from QS, and Hinode/EIS (Brown et al. 2008), which presents data for a QS region and two ARs.

The spatial pixel size for SUMER and EIS is $1^{\prime \prime}$, while it is 1'.8 for HRTS. The theoretical resolution is therefore $2^{\prime \prime}$ for SUMER and EIS, but in reality potentially worse. The reported intensities have accuracies of roughly $\pm 25 \%$.

The inherent horizontal pixel size of our simulation is $47 \mathrm{~km}$, but we choose to rebin to a pixel size equivalent to $1^{\prime \prime}$. We thus increase our pixel size by roughly a factor of 15 in order to reach the theoretical resolution of SUMER and EIS. We must also take photon noise into account. The simulations presented here are noise free, while observations are degraded by a number of noise sources. The following data analysis takes averages over large areas in order to evaluate average Doppler shifts and line widths, but this would lead to wrong conclusions if the same weight were given to low-intensity and highintensity regions. We have therefore imposed a lower limit for the intensities after rebinning to a pixel size of $1^{\prime \prime}$. Locations with lower intensity than a limit of $3 \mathrm{~mW} \mathrm{~m}^{-2} \mathrm{sr}^{-1} \mathrm{~nm}^{-1}$ are disregarded when doing averages over large areas. The value chosen for the limiting intensity is not too important and comes from an estimate of the noise level of both HRTS and SUMER (see Figure 3).

\section{LINE INTENSITY}

The collection of the intensities of the spectral lines from Table 1 are presented in Figure 3. In the same figure, we have plotted the synthetic SE intensities from the initial snapshot of the simulation, calculated using contribution functions from CHIANTI as described in Section 3, in blue. Each data point represents the peak intensity of the spectral line averaged over the full numerical box (FNB).

The intensities fit reasonably well, with a few noticeable exceptions: The observed Fe $\mathrm{XII}_{19.5}$ and $\mathrm{He}_{\mathrm{II}} 25.6$ intensities in the QS observed with EIS by Brown et al. (2008) are much larger than those produced by Bifrost. There can be at least two reasons for this discrepancy. The initial snapshot from which these intensities are produced is cool - the corona is only a few hundred thousand kelvin, so the intensities of the spectral lines with high formation temperature should be lower than what is observed. This should, however, not produce an effect on the $\mathrm{He}_{\text {II }} 2.6$ intensities as that line has a low formation temperature. On the other hand, looking through EIS data, we have not been able to reproduce the intensities quoted by Brown et al. (2008). In fact, picking a few EIS QS data sets at random (eis_10_20100609_071320 and eis_10_20130429 225712 taken at 07:13:20 UT on $2010 / 06 / \overline{09}$ and $22: 57: \overline{1} 2$ on 2013/04/29, respectively), and computing intensities using the EIS software routines EIS_$\mathrm{PREP},{ }^{4}$ we find measured QS intensities that are an order of magnitude lower for both the Fe $\mathrm{XII}_{19.5}$ and $\mathrm{He} \mathrm{II}_{25.6}$.

Assuming that Brown et al. (2008) have by accident found an EIS data set that includes abnormally large $\mathrm{Fe}$ and $\mathrm{He}$ line intensities, there is still a discrepancy between the synthetic intensities for both the Fe line and the Si lines. As discussed below, this discrepancy could be giving information on solar TR and coronal abundances and can be used to derive new "coronal" abundances. Model intensities using these new abundances fit within a factor of a few for all lines, except for the optically thick $\mathrm{C}_{\mathrm{II}_{133.4}}$, which in fact is well reproduced when calculated taking radiative transfer into account (B. Rathore \& M. Carlsson, in preparation).

\subsection{Abundances}

Originally, we started analysis using the standard CHIANTI abundance set of Grevesse \& Sauval (1998). However, it is well known that solar abundances are not always well constrained. A number of uncertainties crop up when deriving abundances in the solar corona and indeed even in the photosphere. In the TR and corona low-FIP elements tend to be overabundant (Laming 2004). In the photospere, synthesized observations based on $3 \mathrm{D}$ simulations indicate that the standard oxygen abundance may be too high (see the discussion of the oxygen abundance in Asplund et al. 2009; Pereira et al. 2013a). We have therefore chosen to use recent estimates for iron (Schmelz et al. 2012), oxygen (Asplund et al. 2009), and silicon (Feldman 1992) abundances as opposed to the standard CHIANTI photospheric abundances. The values used are shown in Table 2, which also shows the references from which they are taken. These choices are made in order to obtain the best possible line ratios between oxygen and silicon lines, which were off by up to a factor 5 when choosing the Grevesse \& Sauval (1998) abundances. Figure 5 shows the line ratios using this paper's selected abundances in the left panel and the ratios using the default CHIANTI abundances in the right panel, both compared with observed ratios. Using the CHIANTI abundances gives ratios that are at least three times lower than what is observed for all line ratios

\footnotetext{
http://solarb.mssl.ucl.ac.uk
} 


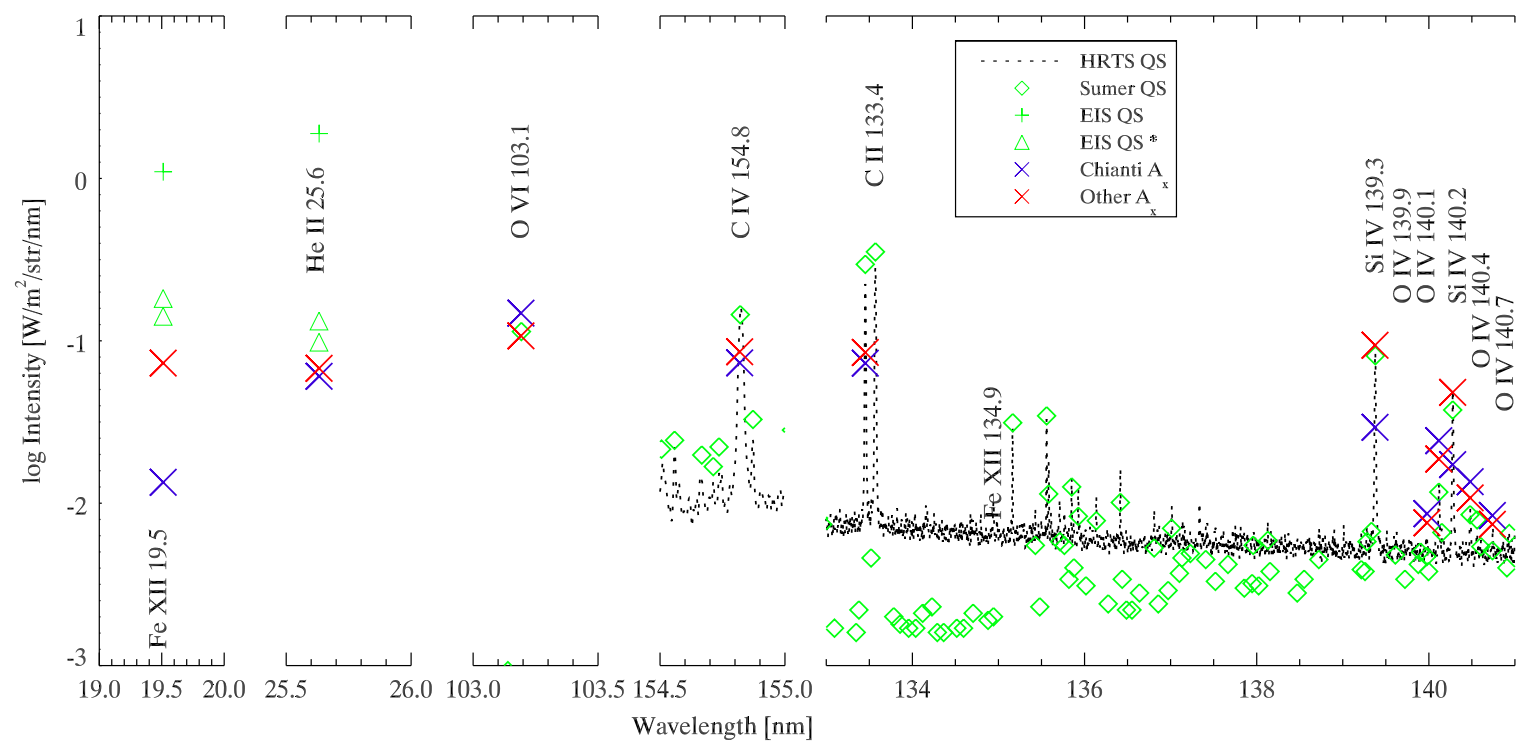

Figure 3. QS intensities from the HRTS atlas (dashed line), the SUMER atlas (green diamonds), Hinode/EIS from Brown et al. (2008) (green crosses), and recalculated Hinode/EIS (green triangles), together with synthetic intensities from the initial snapshot of the simulation with two different abundances (blue and red crosses).

Table 2

Selected Abundance Values

\begin{tabular}{lccl}
\hline \hline Element & CHIANTI $\mathrm{A}_{x}$ & ${\text { New } \mathrm{A}_{x}}$ & $\mathrm{~A}_{x}$ source \\
\hline $\mathrm{Fe}$ & 7.5 & 7.85 & Schmelz et al. (2012) \\
$\mathrm{O}$ & 8.83 & 8.69 & Asplund et al. (2009) \\
$\mathrm{C}$ & 8.52 & 8.59 & Feldman (1992) \\
$\mathrm{Si}$ & 7.55 & 8.10 & Feldman (1992) \\
$\mathrm{He}$ & 10.93 & 10.98 & Feldman (1992) \\
\hline
\end{tabular}

Note. The CHIANTI abundances are from Grevesse \& Sauval (1998), while the source of the others is written in the fourth column.

except the ratio of $\mathrm{Fe} \mathrm{XII}_{19.5}$ to $\mathrm{He}_{\mathrm{II}} 5.6$, which fits well independently of the two choices of abundances presented here. Figure 4 shows the ratio between synthetic and observed QS intensity for all the lines in Table 1. All lines show good agreement with observations. This is also the case in the last 10 minutes of the simulation, when the upper atmosphere heats up considerably. Note that our choice of abundances gives absolute intensities and line ratios in reasonable agreement with the observations.

\subsection{Regional Dependence of the Intensity}

TR lines are well known for the enormous variation they show in intensity from region to region. Presumably this variation depends on the field strength and topology of the magnetic field that reaches the corona. To illustrate some of the issues coupling the field structure and the line intensity of TR lines, we include a discussion of two isolated and magnetically different regions in the following. We consider three different cases: the FNB; a "footpoint region" located just above one of the pores in the photosphere, where the magnetic field is mainly vertical; and an "apex region" located between the two main polarities, where the magnetic field is nearly horizontal.

Figure 6 shows the intensity of the $\mathrm{Si}_{\mathrm{IV}} \mathrm{139.3}_{3}$ line at the beginning of the simulation in the left panel and after 23 minutes in the right panel, with the borders of the footpoint and apex regions superimposed on the images along with a number of field lines in green to indicate the overall topology of the magnetic field. In both panels, the intensity structures follow the overall topology of the magnetic field. The Si IV ion is only present in a narrow temperature interval, the intensity scales closely with the electron density, so the structures in intensity are largely caused by density variations at the formation temperature of the $\mathrm{Si}_{1} \mathrm{IV}_{139.3}$ line, while changes in temperature work like an on/off switch. The intensity of the $\mathrm{Si}_{\text {IV }} 139.3$ line changes between the two images in Figure 6. Initially the intensity is, on average, $94 \mathrm{~mW} \mathrm{~m}^{-2} \mathrm{sr}^{-1} \mathrm{~nm}^{-1}$, while it increases to $279 \mathrm{~mW} \mathrm{~m}^{-2} \mathrm{sr}^{-1} \mathrm{~nm}^{-1}$ in the right panel. That is due to the raised electron density, which is comparatively low in the cool initial snapshot, while the average electron density has increased by a factor of 4 in later snapshots. The intensity has increased most in the apex region, but some of that is due to the especially bright structure that can be seen in the right panel. The intensities of the late snapshot can also be seen in Figure 7. Here, the intensities of the three regions are compared with relevant AR observational data. Almost all lines fit the data very well, especially if we again assume that Brown et al. (2008) have selected an uncharacteristically active data set, and we compare with the data discussed in Section 5.

When we plot the intensity as a function of magnetic field inclination, we find that regions of vertical magnetic field have higher intensities than those that are closer to horizontal: the Probability Density Function (PDF) produced by the intensity of the Si IV line in each data point in the right panel of Figure 6 as a function of the angle of the magnetic field inclination at the formation temperature of the line is presented in the top panel of Figure 8. The lower the formation temperature of the line, the larger the effect of the magnetic field becomes, so much so that the $\mathrm{C}_{\mathrm{II}_{133.4}}$ line has an intensity that is a factor of 60 higher in regions of vertical magnetic field.

Similarly, we investigate the magnetic field inclination dependency of the Doppler shift of each line. Not surprisingly, all lines exhibit, on average, zero Doppler velocity at horizontal magnetic field inclination. The lower TR lines are increasingly redshifted with inclination of the magnetic field up to some 


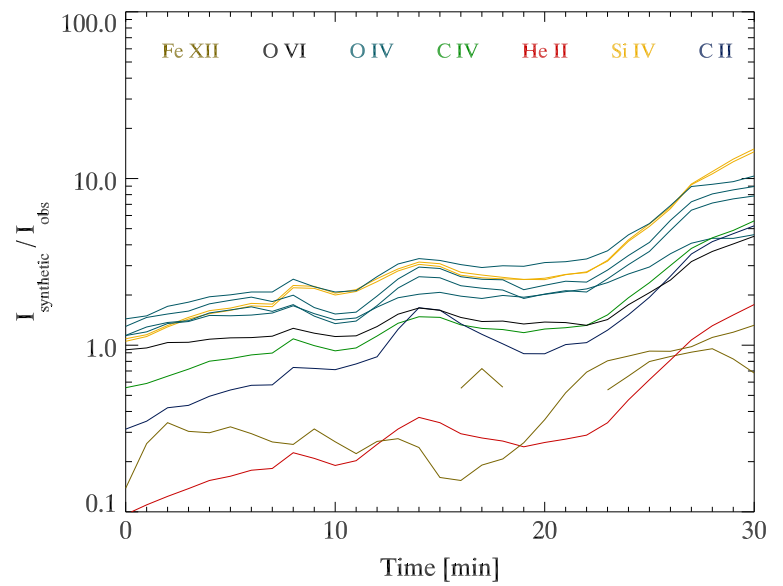

Figure 4. Plot of the ratio between the synthesized spectra and measured QS intensities from observations, where the measured QS intensities are average values from observations, between HRTS and SUMER atlas data for all lines except the Fe XII and He II, where we average between the Brown et al. (2008) data and our two values.

cutoff of $\approx 80^{\circ}$, continuing with decreased redshifts at the most vertical inclination. The hotter $\mathrm{O}$ VI and Fe XII lines, on the other hand, behave differently, showing increased blueshifts as the magnetic field orientation becomes more vertical.

\section{LINE WIDTHS}

The broadening of a spectral line has two causes: atmospheric dynamics and the temperature in the region of the emitting material. The temperature broadens a line owing to the distribution in velocity of the emitting particles, while on larger scale plasma motions cause Doppler shifts, which contribute to the broadening of the line when there are variations in the large-scale velocities along the line of sight. When observing, the width of the line is split into two components: the thermal and nonthermal line width. The thermal line width of a spectral line is given by Equation (4), where the temperature $T_{F}$ is the temperature where the ionization fraction is at its maximum assuming SE. For ions in SE, that is a very good approximation, and if the line is wider than this, it is evidence for processes able to widen the line that are not due to the temperature of the emitting gas. In reality, there can be cases where there is very little or no plasma at that temperature, resulting in most of the intensity coming from ions having a lower temperature, and consequently the line has a peak formation temperature $T_{F}$ that is lower than the temperature where the ionization fraction has its maximum. For the interpretation of line widths, the consequence of such a scenario could be that the nonthermal line width becomes negative because the line is assumed to be formed at the temperature where the ionization fraction has its maximum. This is often seen at full resolution in the simulation described here, but such widths disappear when coarsening the resolution to $1^{\prime \prime}$.

Figure 9 shows the nonthermal line widths for all the lines under consideration as a function of time. We calculate a line width for each of the vertical lines of sight and perform an intensity-weighted average to get a value for the nonthermal line width presented. Behind that average hides a distribution of line widths, with an associated standard deviation. The average nonthermal line width and the standard deviation are shown for the three different locations. The nonthermal line widths for the silicon, oxygen, and carbon lines are fairly constant over long periods of time. The FNB hides events that are able to increase the line widths suddenly, but these are easily visible in the apex and footpoint data, seen as sudden and violent jumps, most prominently in the oxygen lines. The oxygen lines also show a large spread in the distribution of values for the nonthermal line width. In general, the oxygen lines have a standard deviation in their line widths that is as large as or even larger than the average value. The silicon and carbon lines seem to have a narrower distribution. Nonthermal line widths are largely smaller for the footpoint region than for the FNB or for the apex region. The average nonthermal width also seems to increase with formation temperature, with the coldest line formed by $\mathrm{C}_{\mathrm{II}}$ at $\log T=4.65$ having $\xi \sim 8 \mathrm{~km} \mathrm{~s}^{-1}$, increasing to the hottest lines of Fe XII that has $\xi \sim 15 \mathrm{~km} \mathrm{~s}^{-1}$. There are data missing for the hot Fe XII lines in the first 20 minutes, because the intensity does not become large enough to get above our imposed lower limit of $3 \mathrm{~mW} \mathrm{~m} \mathrm{sr}^{-1} \mathrm{~nm}^{-1}$.
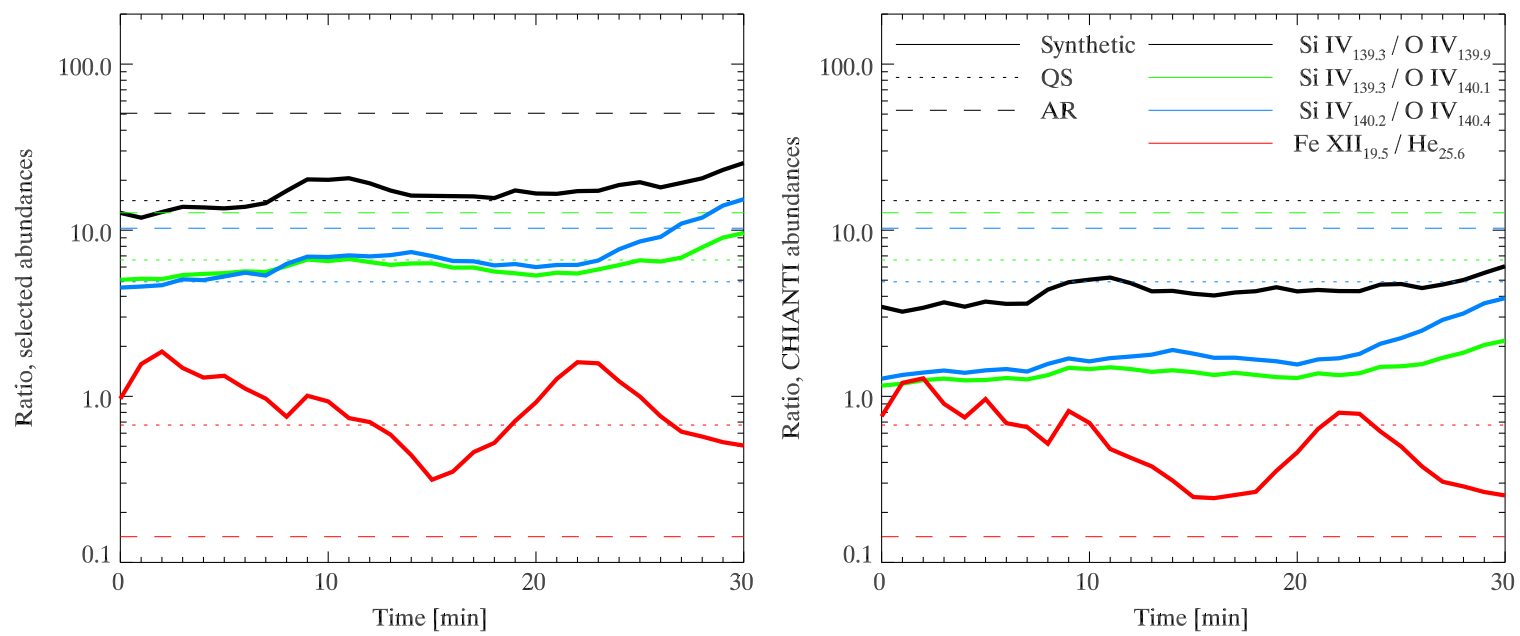

Figure 5. Intensity line ratios, where the thick lines represent the line ratios from the synthetic data, and the dotted and dashed lines represent the line ratio from the average QS and AR data, respectively. The atlas AR data for the $\mathrm{Si} \mathrm{IV}_{139.3} / \mathrm{O} \mathrm{IV}_{139.9}$ ratio is 50.6, which is outside the bounds of the plot. The left panel show the line ratios after we have adjusted the abundance values, while the right panel shows the line ratios when using the Grevesse \& Sauval (1998) abundances. 

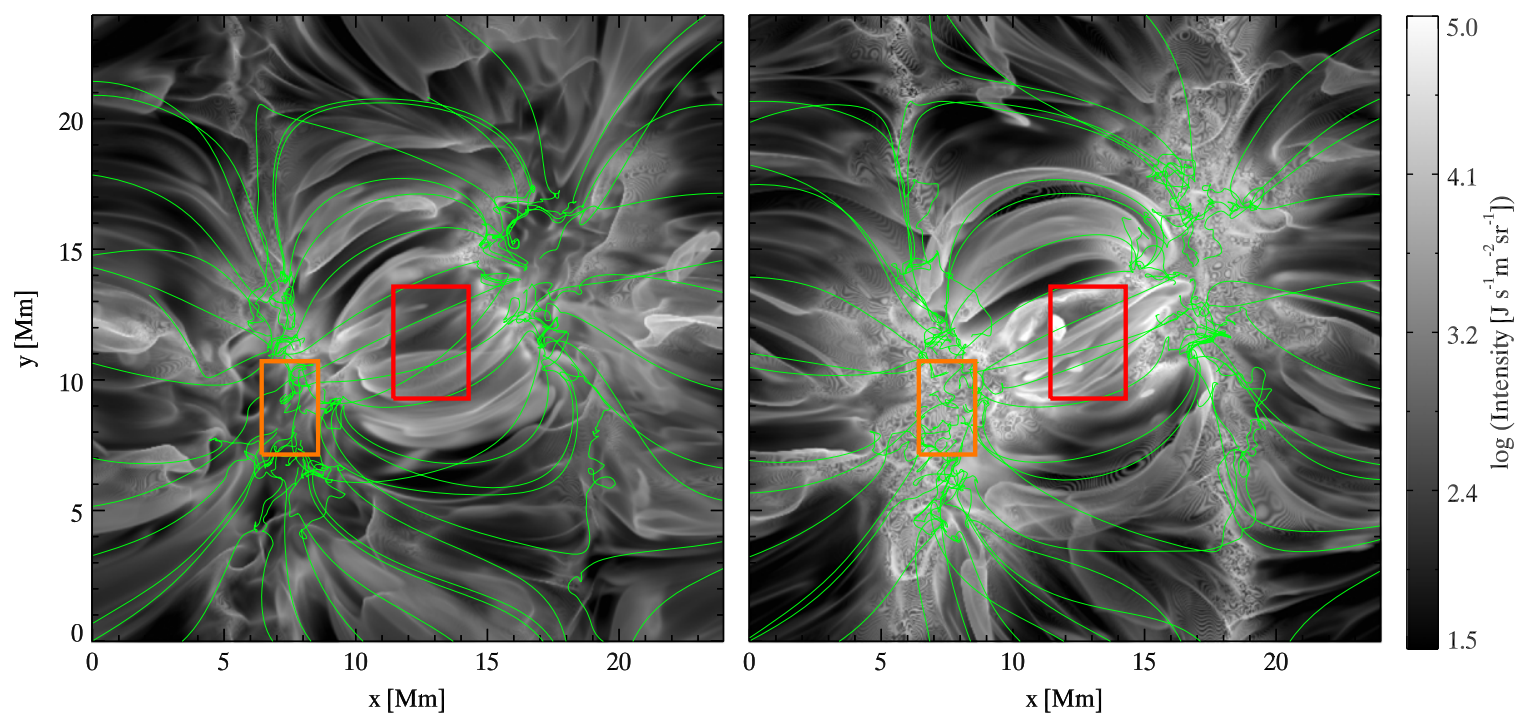

Figure 6. View of the synthetic peak intensity of the $\mathrm{Si}_{\mathrm{IV}_{139} \mathrm{y}_{3}}$ line for the FNB, for the initial snapshot in the left panel and the snapshot 23 minutes later in the simulation in the right panel, with the green lines indicating magnetic field lines. The orange box on the left indicates a region of high magnetic concentration, a footprint region for the magnetic field lines, and vertical field alignment. The red box in the middle indicates a region over magnetic loop apex, where the field is horizontal. The average intensity within the orange and the red boxes from the right panel is presented in Figure 7 by crosses in similar color.

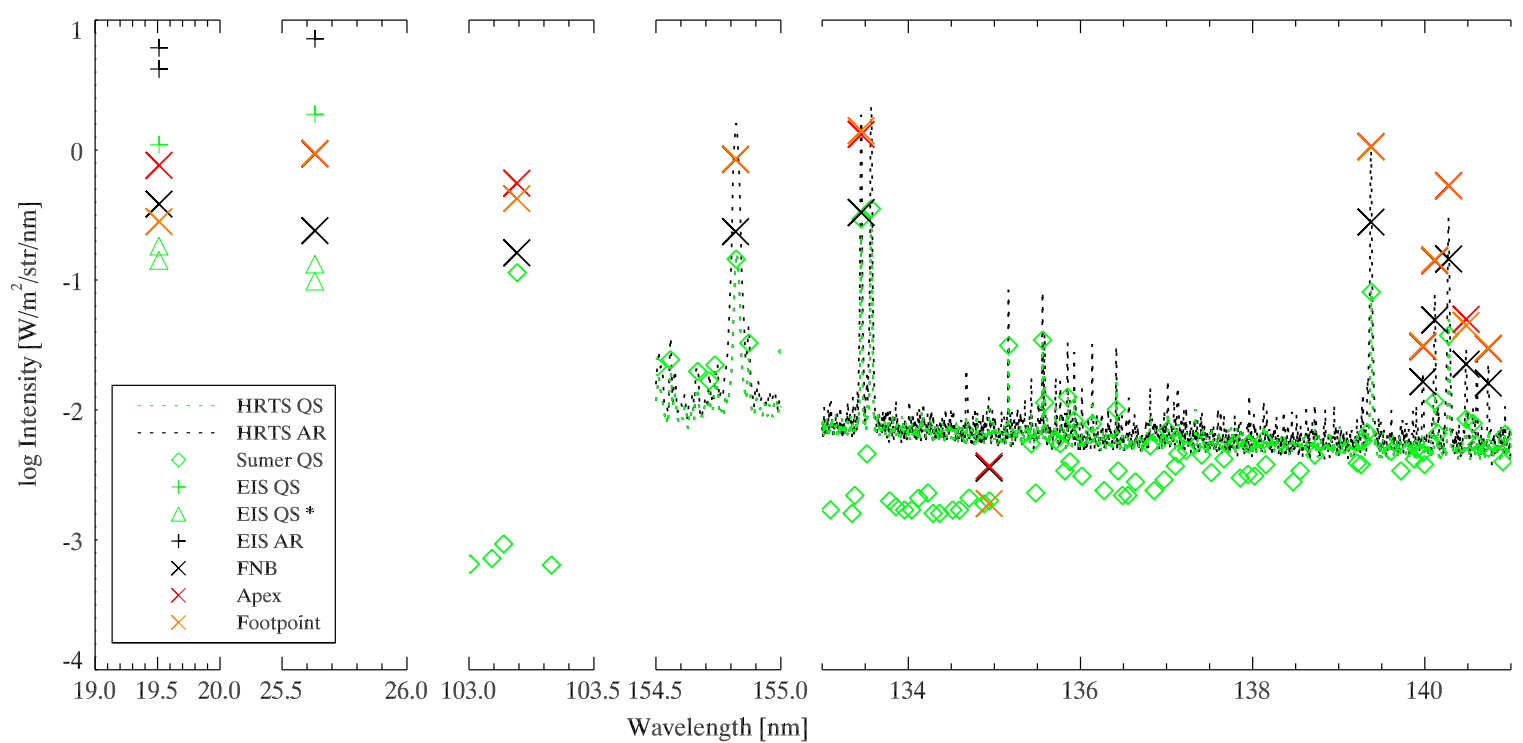

Figure 7. Intensities from the HRTS QS atlas, the SUMER atlas, and Hinode/EIS, together with synthetic intensities from a snapshot 23 minutes after the simulation is initiated. The synthetic intensity values are from averages of the entire FNB in black, while the apex and footpoint regions in red and orange, respectively, are average values within the area of similar color in the right panel of Figure 6.

\subsection{Comparison with Observations}

We have compared observational data compiled by Wilhelm et al. (2007) with the nonthermal line widths from our simulation in Figure 10. We have chosen to plot the values for a single point in time at $t=23$ minutes, as we would like to have a value for the $\mathrm{Fe} \mathrm{XII}_{134.9}$ line included in the plot, while the nonthermal line widths for the other lines are largely constant throughout the whole simulation. There is a difference in the nonthermal line width for the two iron lines. This is due to the fact that the $\mathrm{Fe} \mathrm{XII}_{134.9}$ line is so much weaker than the Fe XII 19.5 line and is below the intensity threshold for most of the FNB. However, the $134.9 \mathrm{~nm}$ intensity is high above the apex region, where we see an agreement between the lines.

The first conclusion that can be drawn from the observed data compiled by Wilhelm et al. (2007) is that the spread in the measured values is large-more than $10 \mathrm{~km} \mathrm{~s}^{-1}$ - similar to the large standard deviation of our simulation. At the same time, the values that come out of our simulation lie below or at best toward the lower end of the QS observational results, and with a lower value for the $\mathrm{O}$ Iv lines at $T=1.6 \times 10^{5} \mathrm{~K}$. There is also an effect of the region we are averaging over: the large FNB shows larger nonthermal velocities, which hints that an effect of spatial resolution is active. In order to investigate this, we repeated the calculation with the pixel size increased to $2^{\prime \prime}$. The larger pixel size increases the nonthermal widths by $11 \%$ on average for lines with formation temperature between $\log T=4.8$ and $\log T=5.5$, for some lines as much as $20 \%$, while hardly changing widths for lines formed outside of this temperature range. There are even claims that the true resolution of SUMER is only 4" (see Kim et al. 2006), so 


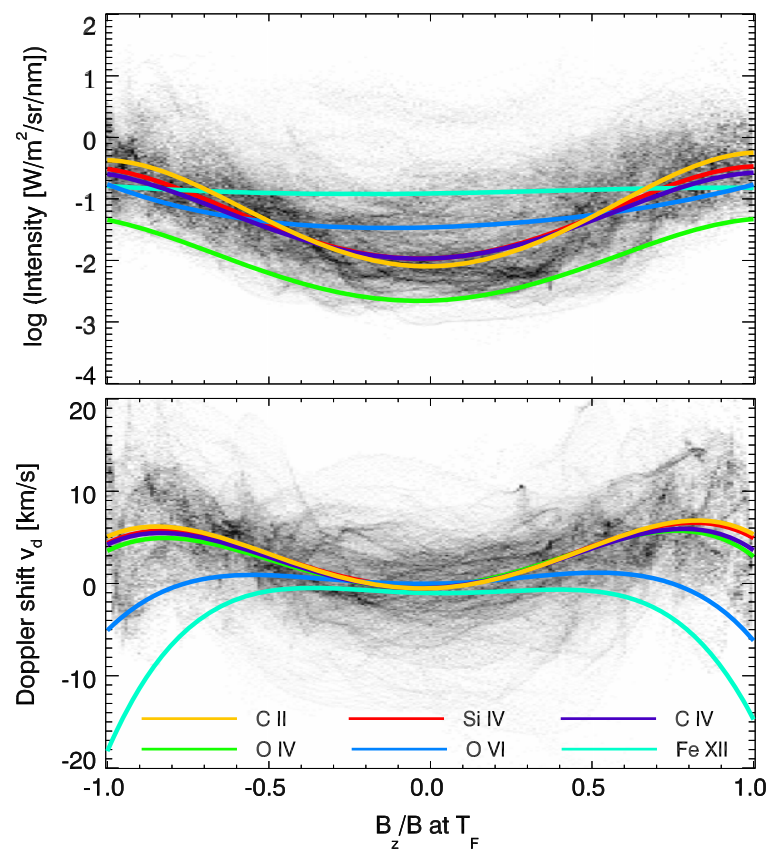

Figure 8. Intensity (top panel) and Doppler shift (bottom panel) as functions of the vertical magnetic field orientation at the formation temperature of the ions, where 0 shows where the magnetic field is purely horizontal and 1 shows where the magnetic field is purely vertical. The intensity seems to follow a quartic function, with the dip of the curve deepening with decreasing formation temperature. The results are the same when the magnetic field is weighted with the emissivity of the spectral lines. For horizontal magnetic field orientation the Doppler shifts are on average zero for all lines, while the lower TR lines show increased redshifts with inclination up to $\approx 80 \%$, while the upper TR lines show increased blueshifts with inclination of the magnetic field.

we could assume even larger values when comparing with SUMER data. However, our computational domain is not large enough to do such smearing without losing statistics, as we then would have only 8 by 8 points left in the subdomain. The results in Figure 9 therefore need to be seen as a lower limit of what SUMER would have observed.

To further demonstrate the effect of spatial resolution, we also plot average values of nonthermal velocities using a spatial resolution similar to that reported by Chae et al. (1998), convolving the data with a $1^{\prime \prime} \times 2^{\prime \prime}$ Gaussian point-spread function (PSF), followed by $3^{\prime \prime}$ smoothing of the spectral profile. This results in synthetic data with a $1^{\prime \prime} \times 3^{\prime \prime}$ spatial resolution. These results are shown as diamond symbols in Figure 10. The increase in the nonthermal line widths resulting from the PSF and the increase of bin size is clearly visible.

Note that the line width for $\mathrm{He}_{\mathrm{II}} 25.6$, formed at $T_{F}=80 \times 10^{4} \mathrm{~K}$, is large. An explanation for this is found when considering the contribution function $(G(T))$ of this line. When compared with the other lines formed at a similar temperatures, it is evident that the normalized $G(T)$ for $\mathrm{He}$ II has contributions from a much wider span in temperature than the other lines, such as those from $\mathrm{C}$ IV or O IV; thus, the helium line samples plasma with a greater range in physical parameters and therefore has greater average width. This reasoning may also explain the generally decreasing line widths as a function of time for the TR lines: as the coronal temperatures in the simulation increase, the TR plasmas are, on average, "squeezed" into smaller spatial volumes by thermal conduction, and the lines, on average, sample a smaller range in physical variables.

\section{DOPPLER SHIFTS}

Peter \& Judge (1999) studied Doppler shifts based on data taken with the SUMER instrument. They examined ultraviolet emission line profiles from the chromosphere to the corona, studying the variation of Doppler shift with formation temperature. As previously found by Chae et al. (1998) and Brekke et al. (1997), the TR lines were on average found to show a redshift. A maximum shift of some $10 \mathrm{~km} \mathrm{~s}^{-1}$ is found for lines formed near $10^{5} \mathrm{~K}$. At greater temperatures the average Doppler shift is reduced and a transition to blueshifts occurs at temperatures of some $3 \times 10^{5} \mathrm{~K}$. Observational data on this topic were collected by Wilhelm et al. (2007) and are included in Figure 11. The observations show a large range in average Doppler shifts, especially for lines formed at high temperature. This reflects a large variability in the regions observed, resulting in the large spread in the reported Doppler widths.

The results of a similar study with the synthetic spectra are plotted together with the observations in Figure 11. As in Section 6, we show the results for a single snapshot at $t=23$ minutes. Similar to what we found for the line widths, we find a large span of Doppler shifts both in the observations and in the simulation. We find a similar picture as in the observations with a net redshift maximum of some $5 \mathrm{~km} \mathrm{~s}^{-1}$ for the FNB and apex data and some $3 \mathrm{~km} \mathrm{~s}^{-1}$ for the footpoint synthetic data. The net redshift decreases with increasing temperature and is in some cases negative for the Fe XII line. Note that the result at high temperature depends on which Fe XII lines are used. This is because the Fe $\mathrm{XII}_{134.9}$ line is so much weaker than the Fe XII 19.5 line; in the cases where the $134.9 \mathrm{~nm}$ intensity is high, there is an agreement between the lines. Both of these lines lie within the acceptable range given by the observations. There is a trend in the Doppler shifts between the different locations. The full FNB seems to, in general, have a bias toward redshifts as compared to the apex region, while the footpoint region has a blue bias compared to the apex region. The footpoint region also has a much larger range in values than the apex and the full FNB.

We also checked our calculated Doppler shifts for the effect of smearing, i.e., the difference between the Doppler shift at the $48 \mathrm{~km}$ native resolution and the new $1^{\prime \prime}$ bin size, but found almost no change in the calculated values, at most $1 \%$ except for the two Fe XII lines. These lines are likely more affected as the number of points that have a significant signal is relatively small, so averaging over a larger area gives relatively large effects; however, it is still only on the order of $10 \%$. On the other hand, applying a PSF to the synthetic data does have an effect. Again following Chae et al. (1998) and using the SUMER PSF as calculated by them, we add plots of the average Doppler velocities to Figure 11 using diamond symbols. An increase in the Doppler velocities resulting from the PSF is clearly seen for all lines considered, except for the Fe $\mathrm{XII}_{134.9}$ line, where the Doppler velocities decrease.

\section{SPECTRAL LINE CORRELATIONS IN THE TR}

In many previous studies of the TR spectra, some form of correlations has been found between any two combinations of the intensity, line width, and Doppler shift. It is therefore instructive to investigate how well the simulated atmosphere produced by Bifrost is able to reproduce these correlations and implicitly tell us how well the simulation holds against the real 

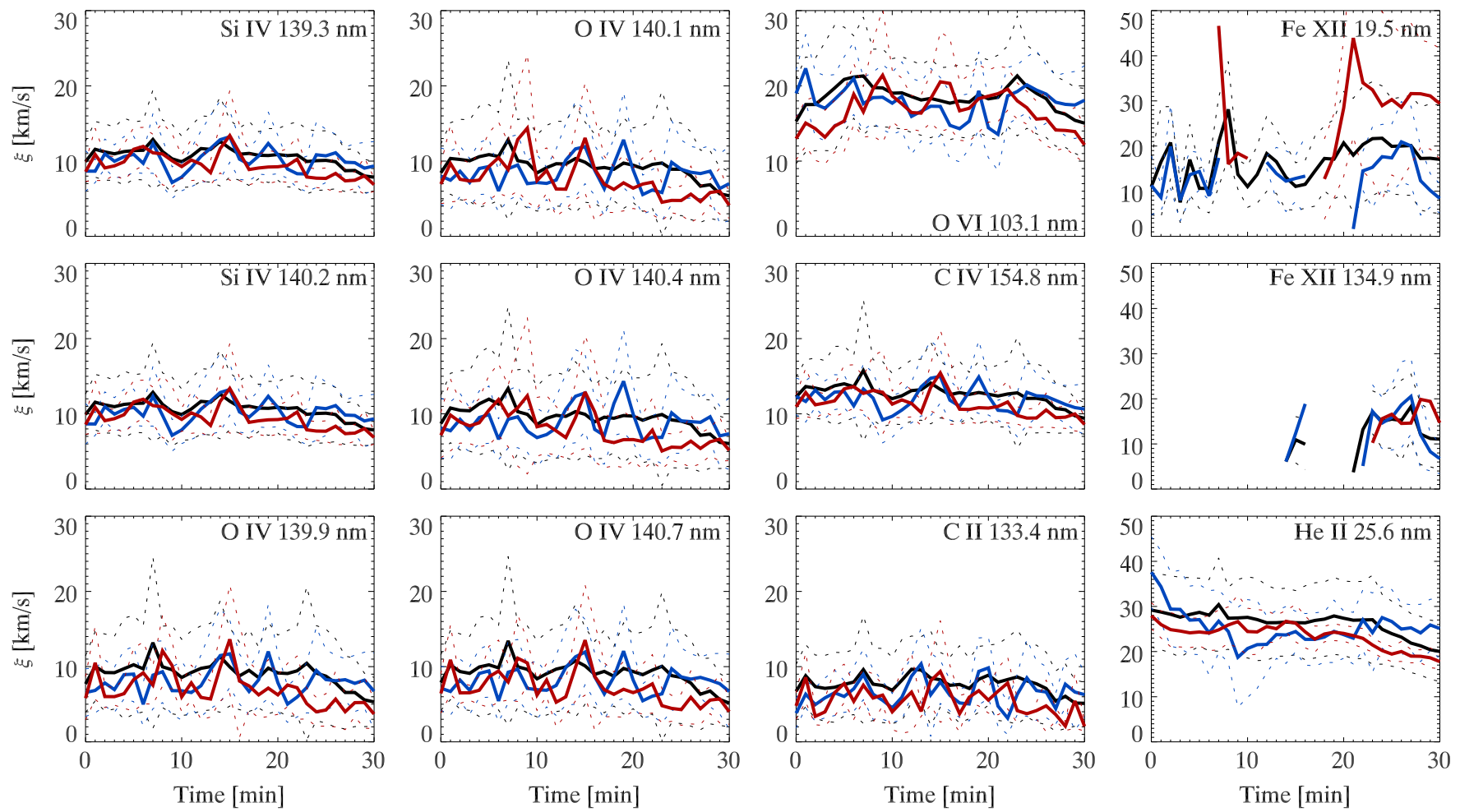

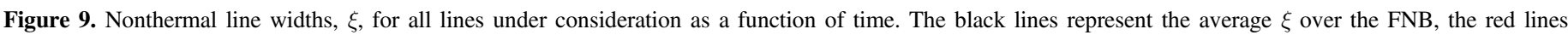

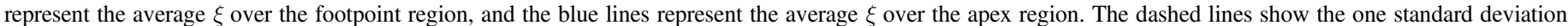
range of the respective region.

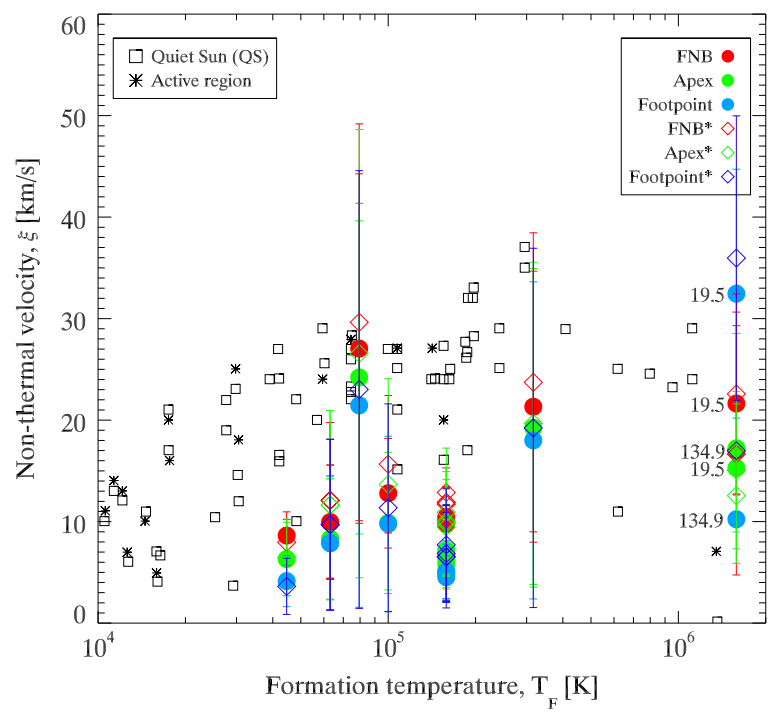

Figure 10. Comparison of the synthetic nonthermal line widths and the observed nonthermal line widths. The circles show the synthetic nonthermal line widths, 23 minutes into the simulation. The green symbols show the results from the FNB data, the red from the footpoint region, and the blue from the apex region. The data points in black show QS and AR results from the comprehensive collection of observational data done by Wilhelm et al. (2007). The diamond symbols represent the same data after applying a PSF and rebinning according to the SUMER spatial resolution reported by Chae et al. (1998).

atmosphere of the Sun. We here investigate some of these correlations by calculating the linear Pearson correlation coefficient between the observables. The correlation coefficient is +1 in the case of a perfect linear relationship and -1 in the case of perfect decreasing linear relationship. As the coefficient approached zero, the correlation between the variables weakened, where zero represents uncorrelated data.

\subsection{Line Width Correlations}

There have been many studies on correlations between the observables done from TR spectra. Chae et al. (1998) reported that there are positive correlations between the intensity and the nonthermal line widths for spectral lines formed in the temperature interval between $2 \times 10^{4}$ and $10^{5} \mathrm{~K}$, with a weakening in correlations with increasing temperature, in addition to finding a positive correlation in nonthermal line widths for a set of any two lines with formation temperature below $2 \times 10^{5} \mathrm{~K}$. Akiyama et al. (2003) confirmed this correlation for the $\mathrm{Si} \mathrm{IV}_{140.2}$ and the $\mathrm{O}_{\mathrm{IV}_{140.1}}$ lines. A good test for the synthesized spectra is to see whether these correlations are reproduced.

In Figure 12 a scatterplot showing correlations for some of the lines in our selection is presented. As demonstrated by these figures, there is little correlation between the intensity and the nonthermal line width for the TR lines, demonstrated by the small correlation coefficients. The Fe XII 19.5 line data, on the other hand, are anticorrelated. After applying the PSF, the correlation seems slightly stronger. Thus, while we may have found a weak correlation between the intensity and nonthermal line width for the TR lines, it is much weaker than what was reported by Chae et al. (1998). The positive correlations found are due to the difference in the PSF size and the subsequent smoothing, from $1^{\prime \prime} \times 2^{\prime \prime}$ to $1^{\prime \prime} \times 3^{\prime \prime}$, respectively, causing spatial coherence in some of the resulting data points, and thus leading to the small but positive correlation in the result. The spatial resolution and photon leakage in the CCD (here represented by the PSF) are therefore important, and one 


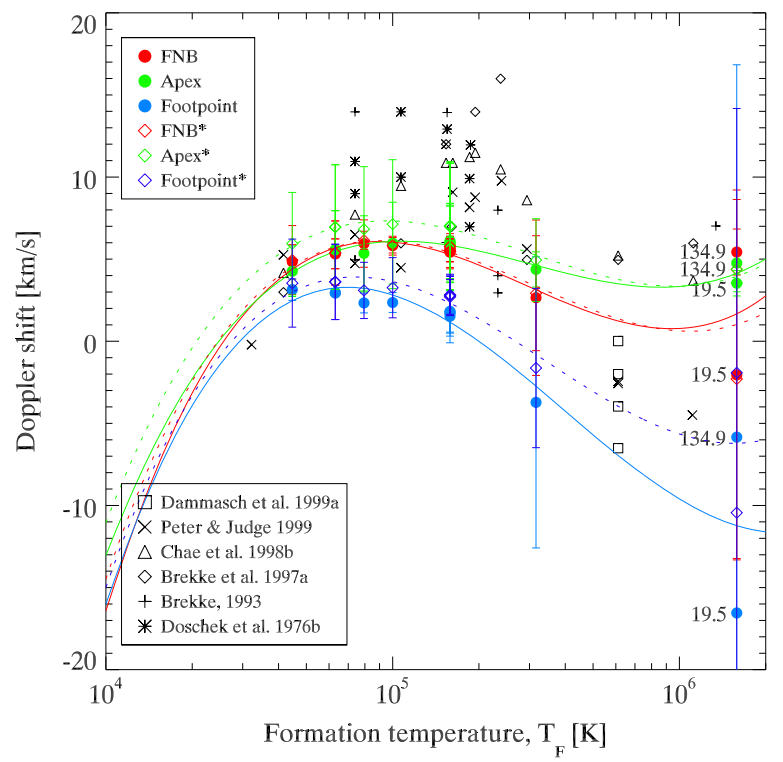

Figure 11. Comparison of the synthetic Doppler shifts and the observed Doppler shifts. The circles show the synthetic Doppler shifts, 23 minutes into the simulation. The green symbols show the results from the FNB data, the red from the footpoint region, and the blue from the apex region. We have interpolated the data set from each region to a third-degree polynomial to help guide the eye. The diamond symbols represent the same native data after applying a PSF and rebinning according to the spatial resolution reported by Chae et al. (1998). The data points in black show the comprehensive collection of data from observations done by Wilhelm et al. (2007).

should therefore be very carful when using correlations of intensity to nonthermal line width between the lines for any diagnostics of the solar atmosphere.

There are strong positive correlations between the nonthermal line widths for the TR lines, with the strongest correlation being between the Si IV and the O IV lines. There is close to no correlation between the Fe $\mathrm{XII}_{19.5}$ and the $\mathrm{C} \mathrm{IV}_{154.8}$ line in the black $1^{\prime \prime} \times 1^{\prime \prime}$ data. The scatterplot for the nonthermal line widths of the $\mathrm{OVI}_{103.1}$ to the $\mathrm{CIV}_{154.8}$ line seems to show two separate populations, one showing positive correlations similar to the other TR lines, and the other showing a $30 \mathrm{~km} \mathrm{~s}^{-1}$ spread in nonthermal line widths of the $\mathrm{O} \mathrm{VI}_{103.1}$ line centered around $10 \mathrm{~km} \mathrm{~s}^{-1}$ in the $\mathrm{C}_{\mathrm{IV}_{154.8}}$ nonthermal line width. This is similar to the correlations of nonthermal line widths of the Fe XII 19.5 to the $\mathrm{C}_{\mathrm{IV}_{154.8}}$ line. Since the $\mathrm{O} \mathrm{VI}_{103.1}$ line is formed high in the TR, an explanation for this can be that we are seeing correlation results of both the TR and the corona. This spread into two populations in the scatterplot is seen in the data set as soon as we have high enough coronal temperatures. In the PSF convolved data this split is not as clear.

The correlation coefficients are highest for the mid-TR lines of Si IV, O IV, and C IV and decrease with temperature, similar to results reported for solar values by Chae et al. (1998). The strong correlation seen between the Si IV and O IV line is also reported by Akiyama et al. (2003).

We conclude that we are not able to produce the observed correlation between the line widths and the intensities of the lines in our selection on the native data. There may be a tendency for a slight correlation after applying a PSF and smoothing the data. However, this is not enough to reproduce the observed weak correlation. The correlations in nonthermal line widths between any two lines formed below $10^{6} \mathrm{~K}$ in our line selection are positive, as reported by Chae et al. (1998).

\subsection{Doppler Shift Correlations}

Doschek (2006) report on Doppler shift correlations in the TR lines using SUMER observations. They find strong correlations for the lower TR lines, but when investigating the correlation between a lower TR line and an upper TR line, the correlation is much weaker. They conclude that the emission arising from the lower TR comes from cool loop structures, while the hotter lines emerge from separate structures.

We have done a similar study on the synthetic emission from the simulation. The Doppler shift to Doppler shift correlation coefficients are listed in Table 3 for the lines under consideration, where we have neglected to list the spectral line of the same ion twice, since they have the same correlation coefficients. The information listed in the table shows, not surprisingly, that we have a strong correlation between spectral lines when the difference in the formation temperature between the lines is small. The correlation weakens as this difference increases, represented by the decreasing correlation coefficient with increasing formation temperature of the lines in the table.

Some of the Doppler shift correlations are plotted in Figure 13. These figures reproduce the study of Doschek (2006), showing strong correlation from the Doppler shifts of the lower TR lines and the decrease in correlation for the upper TR lines. The high correlation coefficients imply a strong relationship within the temperature region below $\log T=5.2$. The correlations are high between the lower TR lines and the upper TR line of $\mathrm{O}$ VI as well, and there is clearly a connection between at least some of the $\mathrm{O}$ VI structures and the lower TR structures. Applying a PSF to the data does not change the results.

The correlations between the Fe XII Doppler shifts and the Doppler shifts of the colder lines are very low, including that of $\mathrm{O}$ VI, showing that these lines originate mostly from other structures. The lack of correlation can also be due to different physics dominating the region of emission, such as the strength of thermal conduction. The physical distance between the volume in a loop emitting at the chromospheric and TR line temperatures is small, since the large gradient in temperature makes the vertical distance small and, depending on the magnetic topology, the horizontal distance small as well. This leads to the emitting material at TR temperatures being closely linked, explaining the close correlation between Doppler shifts. The larger the difference in formation temperature, the larger the potential vertical and horizontal distance between the emitting material and consequently a decrease in correlation coefficient.

Doschek (2006) came up with two explanations for these correlations: (1) small loops below the spatial resolution of $1^{\prime \prime}$ that are side by side at different temperatures, e.g., a loop mostly emitting from one ion next to a loop mostly emitting from another ion, or (2) a single loop that is transiently heated, e.g., producing mostly emission from one ion at one time and mostly emission from another ion at another time, the times being significantly less than the exposure time.

While Doschek (2006) favors the second explanation owing to the long exposure times from the solar data and unresolved fine structures (UFSs), we find that in our models both mechanisms are active. As discussed by Guerreiro et al. 

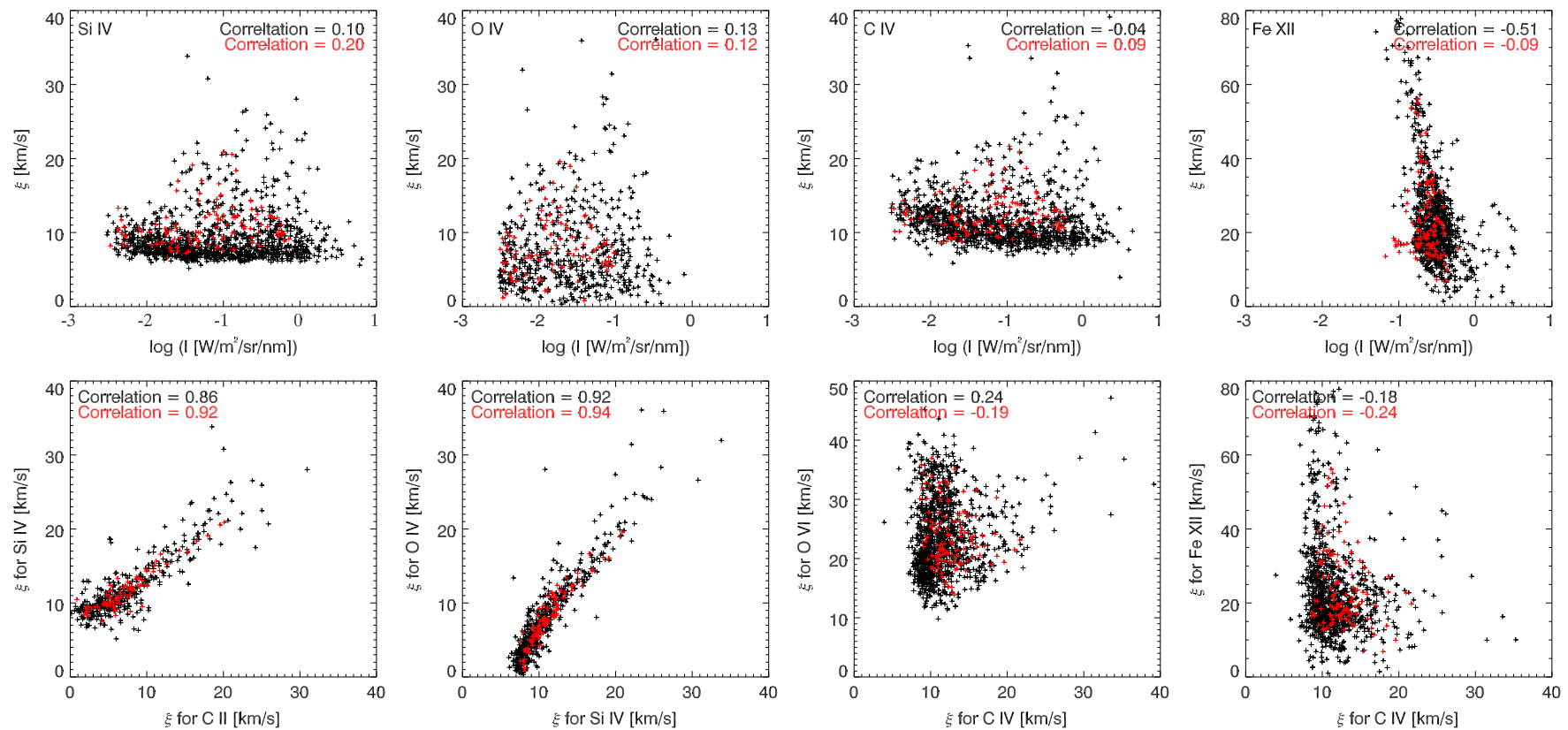

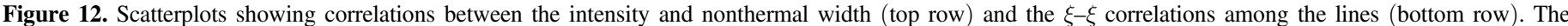

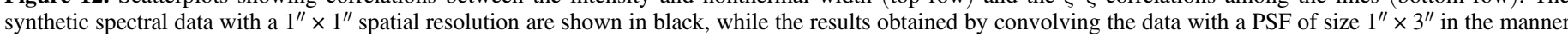
of Chae et al. (1998) are shown in red, for the snapshot 23 minutes into the simulation.

Table 3

Doppler Shift Correlation for the Respective Lines, 23 Minutes into the Run

\begin{tabular}{lcccccr}
\hline \hline Line $[\mathrm{nm}]$ & Si IV & He II & C IV & O IV & O VI & Fe XII \\
\hline $\mathrm{C} \mathrm{II} \mathrm{II}_{133.4}$ & 0.96 & 0.88 & 0.91 & 0.79 & 0.62 & -0.07 \\
$\mathrm{Si} \mathrm{IV}_{139.3}$ & $\ldots$ & 0.93 & 0.97 & 0.96 & 0.69 & -0.04 \\
$\mathrm{He} \mathrm{III}_{25.6}$ & $\ldots$ & $\ldots$ & 0.98 & 0.98 & 0.81 & -0.01 \\
$\mathrm{C} \mathrm{IV}_{154.8}$ & $\ldots$ & $\ldots$ & $\ldots$ & 0.99 & 0.76 & -0.01 \\
$\mathrm{O} \mathrm{IV}_{140.1}$ & $\ldots$ & $\ldots$ & $\ldots$ & $\ldots$ & 0.85 & 0.06 \\
$\mathrm{O} \mathrm{VII}_{103.1}$ & $\ldots$ & $\ldots$ & $\ldots$ & $\ldots$ & $\ldots$ & 0.41 \\
\hline
\end{tabular}

Note. The lines are listed as a function of increasing formation temperature. Fe XII in the top row represents the Fe $\mathrm{XII}_{134.9}$ line, while the others are the same as the spectral lines listed in the rightmost column, i.e., $\mathrm{C} \mathrm{II}_{133.4}$ to $\mathrm{Si}$ IV represent the $\mathrm{C}_{\mathrm{II}_{133.4}}$ to $\mathrm{Si}_{\mathrm{IV}_{139.3}}$ correlation, and so forth. The results do not change when applying a $2^{\prime \prime}$ PSF on the data.

(2013), loops can be sorted into two categories based on the dominant cooling mechanism: cool low TR loops that lose energy through radiative losses, and warmer coronal loops that lose energy via thermal conduction. At any given moment several small TR loops with slightly different temperatures, but otherwise similar physical properties, coexist, favoring explanation 1 . On the other hand, the model shows that these TR loops are far from static and cycle through a range of temperatures in a short time span, favoring explanation 2 . The modeled hotter loops have longer timescales and in general a different spatial distribution than the cool loops and thus have a much smaller correlation with the cooler TR loops.

Doschek et al. (2008) studied correlations between Doppler shifts and nonthermal line width of the Fe XII 19.5 line, over two ARs observed with Hinode/EIS, and found a strong negative correlation.

We performed the same study on the synthetic spectra from our model, for all the lines under consideration. We find no correlation between the nonthermal line widths and the Doppler shifts, except for the iron lines, which are strongly correlated. The correlation is observed starting 20 minutes into the run, just as the Fe XII intensity and nonthermal line widths become sufficiently large to study, with a correlation coefficient varying between 0.5 and 0.8 for the remaining time of the simulation. We are thus able to reproduce the correlation reported by Doschek et al. (2008). Doschek et al. (2008) presented Doppler shifts from 10 to $50 \mathrm{~km} \mathrm{~s}^{-1}$ and nonthermal motions from 20 to $90 \mathrm{~km} \mathrm{~s}^{-1}$. We get significantly lower nonthermal speeds, where most of the data points are below $40 \mathrm{~km} \mathrm{~s}^{-1}$. On the other hand, the Doppler speeds are similar to the observed values, between -50 and $10 \mathrm{~km} \mathrm{~s}^{-1}$. The correlations, Doppler shifts, and nonthermal widths are similar for the Fe XII 134.9 line. The results are presented in black in Figure 14.

The physical explanation of this correlation is quite simple, where the small Doppler shifts represent regions of low dynamic activity, which is mirrored by the low nonthermal line widths. As intensity of the dynamics increases, we observe stronger Doppler shifts, where the high dynamic activity is mirrored in the high nonthermal line widths.

By convolving and rebinning the synthetic data in a manner similar to that reported by Doschek et al. (2008), where the data are convolved by a $2^{\prime \prime} \times 2^{\prime \prime}$ Gaussian PSF and rebinned to $1^{\prime \prime} \times 1^{\prime \prime}$, we lose the lowest and highest values of the nonthermal motions, but the effect is marginal.

\section{INTRODUCING NONEQUILIBRIUM IONIZATION}

Owing to the rapidly changing properties of the solar atmosphere, many of the emitting ions of the TR and corona have ionization and recombination timescales longer than the dynamical timescales of the region. This implies that these ions can be found far from their equilibrium temperature and spectroscopic investigations can no longer be trusted to give information of the local quantities. It is therefore important to be aware that assuming SE might lead to incorrect results, and that it is crucial to consider nonequilibrium ionization effects when interpreting observations. Olluri et al. (2013b) developed a nonequilibrium solver for the Bifrost code, which we use 

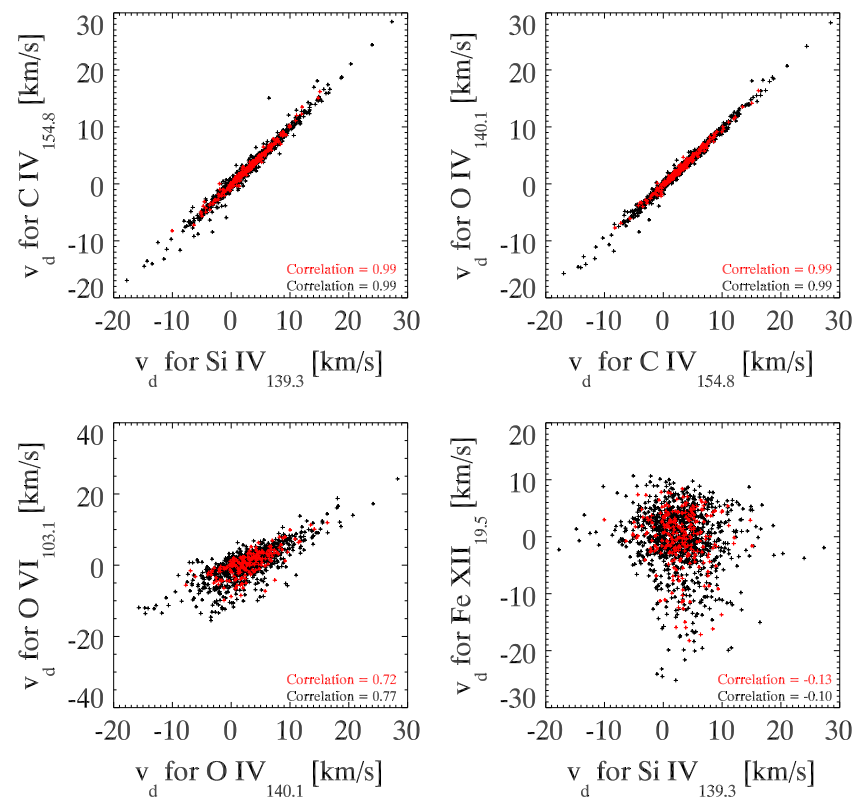

Figure 13. Scatterplots of the spatial correlation of the Doppler shifts between some of the lines, showing increasing correlation with decreasing difference in formation temperature of the lines. The black scatter represents the $1^{\prime \prime} \times 1^{\prime \prime}$ bin

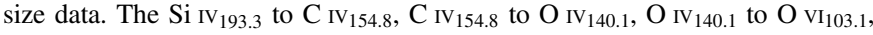
and $\mathrm{Si} \mathrm{IV}_{139.3}$ to the $\mathrm{Fe} \mathrm{XII}_{19.5}$ correlations are plotted in the top left, top right, bottom left, and bottom right panels, respectively. The results do not change when applying a $2^{\prime \prime}$ PSF to the data, represented by the red scatter data.

here, solving the rate equations for some of the elements considered in this paper.

We have constructed atomic models for iron, silicon, oxygen, and carbon atoms using the DIPER package (Judge $\&$ Meisner 1994), and proceed by solving the rate equations as described in Olluri et al. (2013b):

$$
\frac{\partial n_{i}}{\partial t}+\nabla \cdot\left(n_{i} \mathbf{u}\right)=\sum_{j \neq i}^{N_{l}} n_{j} P_{j i}-n_{i} \sum_{j \neq i}^{N_{l}} P_{i j}
$$

where $\mathbf{u}$ is the macroscopic velocity, $n_{i}$ is the population density of ion level $i$, and $P_{i j}$ is the transition rate coefficient between level $i$ and level $j . N_{l}$ is the total number of levels in the model atom.

The equation is solved in two steps using operator splitting. First, the continuity equation on the left-hand side is forwarded in time using a first-order upwind scheme. This solution is then used to solve the time-dependent SE equations, resulting in the $\mathbf{n}^{*}$ advected population densities. The remaining equations are reduced to a linear set $\mathbf{n}=\mathbf{A}^{-1} \mathbf{n}^{*}$ by the optically thin approximation, where we get an $N_{l} \times N_{l}$ rate matrix $\mathbf{A}$ with elements $A_{i i}=\left(1+\Delta t \sum_{j \neq i} P_{i j}\right)$ and $A_{i j}=-\Delta t P_{j i}$. The optical depths are below 1 for all ions investigated here ( $\mathrm{Si}$ IV, C IV, O IV, and FexII), and the approximation holds.

The rate equations are solved for each element separately, at every time step in the simulation, using values of the temperature, advection velocity, and mass density at each grid point. The solution of the rate equations is at all times coupled to the overall dynamics of the synthetic atmosphere but does not affect the solution of the rate equations for the other elements.

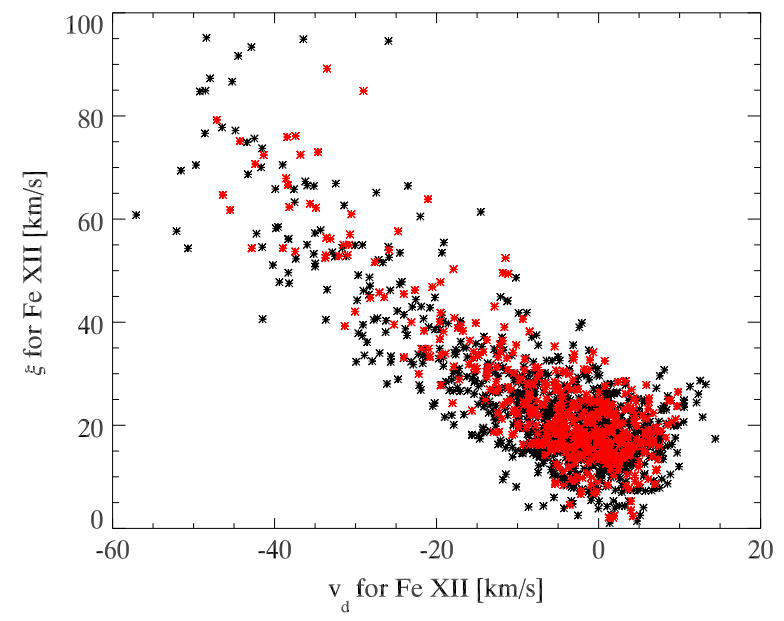

Figure 14. Scatterplot of the Doppler shift with nonthermal line width for the Fe XII 19.5 line 23 minutes into the run, with correlation coefficient -0.82 . The results are strikingly similar to results presented by Doschek et al. (2008), where the only difference is a $30 \mathrm{~km} \mathrm{~s}^{-1}$ shift in the $\xi$ values. The red data points show the results after applying a $2^{\prime \prime} \mathrm{PSF}$.

\subsection{Ions out of Ionization Equilibrium}

Each of the atomic models consists of a number of levels, composed of both ionization stages and excitation levels. The number of levels within each atomic model has been carefully selected using DIPER, in a matter that the ionization balance is kept when the SE equation is solved for arbitrary temperatures and electron densities.

The silicon atom model includes ionization levels I to VI with two extra levels for Si I and Si IV and one extra level for Si II and Si VI, giving a total of 12 levels.

The oxygen atom model includes ionization levels II to VII with two extra levels for $\mathrm{O}_{\mathrm{III}}$ and six extra levels for $\mathrm{O}_{\mathrm{IV}}$, making the oxygen model consist of 14 levels in total.

Our carbon model consists of ionization levels I to $\mathrm{V}$ and has two extra levels for $\mathrm{C}$ III and one extra level for $\mathrm{C}_{\mathrm{IV}}$, giving a total of eight levels.

Finally, our iron model consists of a total of 20 levels, which are the ionization states of Fe x to Fe XV where Fe XII and Fe XIII have seven extra levels each.

Most of the levels in these models are necessary to adequately describe the ionization balance of the ions, while others (especially the iron atom model) have additional levels that have been included for future studies.

We run one simulation for each of the atomic models, solving the rate equations as described in Section 9. After a very short time, the simulations reach a new quasi-equilibrium, which is not to be mistaken for SE for the ions, but rather an equilibrium that results from the ion fractions portraying a relaxation with the new dynamic environment. This quasiequilibrium for the four ions ( $\mathrm{Si}$ IV, O IV, C IV, Fe XII) is plotted as PDFs in Figure 15 in black, where we also have plotted the equivalent PDFs for an SE solution in red to show the significant departure from the equilibrium solution. We concentrate on these ionization stages, because they ( $\mathrm{Si}$ IV, $\mathrm{O}$ IV, Fe XII) are the ones emitting in the IRIS passband, while the $\mathrm{C}$ IV emission has been thoroughly studied by previous space missions.

In general, the ions presented in Figure 15, except for Fe XII, are present in a temperature interval much larger than for statistical equilibrium. The dynamical behavior in the TR 

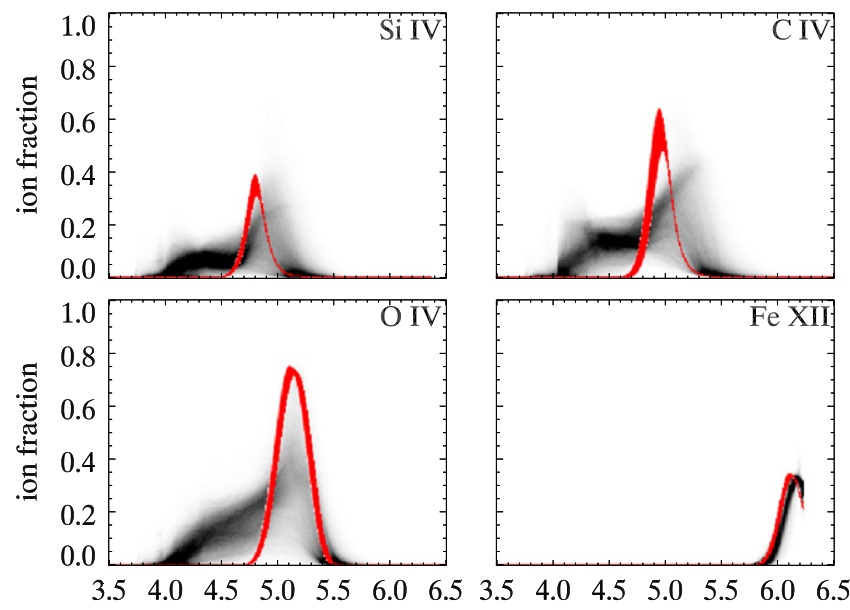

$\log \mathrm{T}[\mathrm{K}]$

$\log \mathrm{T}[\mathrm{K}]$

Figure 15. PDF of ionization fractions as a function of temperature for the ions of interest. The black PDF shows the NEQ distributions, while the red shows what the distributions would be under SE conditions.

causes changes in the local quantities of the plasma, where advection through temperature gradients is common, leading to rapid changes in the plasma temperature and density. If the ions have long ionization and/or recombination times, the SE distribution cannot be maintained when the plasma is rapidly cooled or heated, as, for instance, when the plasma is advected up through the transition zone. When investigating the gas temperature, the emission of the ions will therefore seem to originate from temperatures lower than their SE temperatures. That can be even more severe if the recombination rate decreases rapidly with temperature, as for $\mathrm{Si}$ IV. If the plasma is rapidly heated and the ionization rate is low, the lowtemperature ions can be accumulated even though the plasma has a much higher temperature. The result is a much larger range of temperatures that the ions can be found in.

In the models presented here iron is very near SE. There are small deviations, but the conditions for bringing Fe XII out of equilibrium are not present. A hotter, more dynamic lower corona would be able to bring iron out of $\mathrm{SE}$, as has been shown in Olluri et al. (2013b) in the case of a rapid heating.

One could argue that even though the ions are out of ionization equilibrium, most of the emission can still originate from similar regions to those in the SE case. We have therefore plotted the emissivity of the $\mathrm{Si}_{\mathrm{IV}_{139.3}}, \mathrm{C} \mathrm{IV}_{154.9}, \mathrm{O}_{\mathrm{IV}} \mathrm{I}_{139.3}$, and $\mathrm{Fe} \mathrm{XII}_{19.5}$ lines as functions of temperature in the four panels of Figure 16. As the PDFs demonstrate, the emissivity distribution is wider, where the emission from the hotter side of the distribution is similar to the SE results, but stretches to lower temperatures than does the SE. For all TR lines, the peak emissivity is much lower in NEQ then in SE, but spread over a larger temperature region.

We now take a closer look at the synthetic spectra produced when NEQ effects are included, for the spectral lines listed in Table 1 emitted from the four ions discussed here. For this we again coarsen the pixel size to an equivalent of $1^{\prime \prime}$ and calculate the emissivity according to Equation (1), where $n_{u}$ is the upper level of the transition found by solving the rate equations, and then continue by solving Equations (6)-(9) to retrieve the synthesized spectra.
It should be noted that the rates in DIPER are not as up-todate as in CHIANTI, but the flexibility of DIPER in creating the appropriate atomic models for studies such as this makes the package an essential necessity. Ideally, we would like to have used the most recent data included in CHIANTI, but we were not able to find the references or values for many of the rate coefficients used in CHIANTI from the CHIANTI manual. Especially the rates for the iron lines are very different from the DIPER rates. This makes it impossible to make a direct comparison between the intensity, line width, and Doppler shifts from the previous sections, where the emissivity was calculated using CHIANTI contribution functions (following Equation (2)) to emissivity calculations following solutions of the SE equations of the atomic models (following Equation (1)).

In Figure 18 we have plotted the time evolution of the average intensities, nonthermal line widths, and Doppler shifts synthesized using SE solutions and the nonequilibrium results.

\subsection{NEQ Abundances}

As in Section 5.1, we look at the line ratios from the simulation and see how they compare with the observed line intensity ratios using different abundance values. These are plotted in Figure 17, showing the line ratios over a 20minute time interval. As the values in the table show, the line ratios using the new abundances listed in Table 2 reproduce observed values, while the CHIANTI abundances result in line intensity ratios that are not compatible with the intensity line ratios for the different atlases. The abundances in SE reproduced the line ratios fairly well, but here we see that the NEQ effects change the ratios, and we conclude that derived abundances could be affected by NEQ effects. The silicon-tooxygen ratios presented here give values that are $10 \%-20 \%$ higher in NEQ than in SE depending on the noise limit imposed, the exact time of the observation, and the specific line ratio, leading to an increase in the uncertainty of observationally determined abundances.

\subsection{NEQ Intensity}

The time evolution of the average intensity over the FNB, apex region, and footpoint region in both SE and NEQ is plotted in the top row in Figure 18, for the $\mathrm{Si} \mathrm{IV}_{139.3}, \mathrm{C}_{\mathrm{IV}_{154.9}}$, and $\mathrm{O}_{\mathrm{IV}} \mathrm{I}_{140.1}$ lines, respectively. We have chosen to only show the results for one spectral line of each ion since the general behavior of these is representative for the other spectral lines of the ions here. The plot shows that the NEQ intensities over the FNB and the footpoint region are higher than equivalent SE results throughout the time series. On the other hand, for the apex region this seems to vary. On average, the FNB and apex intensities increase by $\approx 10 \%$ while the footpoint intensity increases by $20 \%$ for the O IV lines when including nonequilibrium ionization. For the Si IV lines, the FNB, apex, and footpoint intensities increase by $\approx 40 \%, \approx 10 \%$, and $\approx 30 \%$, respectively. The $\mathrm{C}_{\mathrm{IV}}{ }_{154.9}$ line intensity is increased by $\approx 50 \%$, $\approx 20 \%$, and $\approx 70 \%$ over the FNB, apex region, and footpoint region, respectively. The Fe XII intensities change by only a few percent, and since the differences are so small, they are not included in the figure.

After only 3 minutes or so the equilibrium ionization solution is lost and a quasi-steady ionization state is reached that remains virtually unchanged until the end of the simulation 

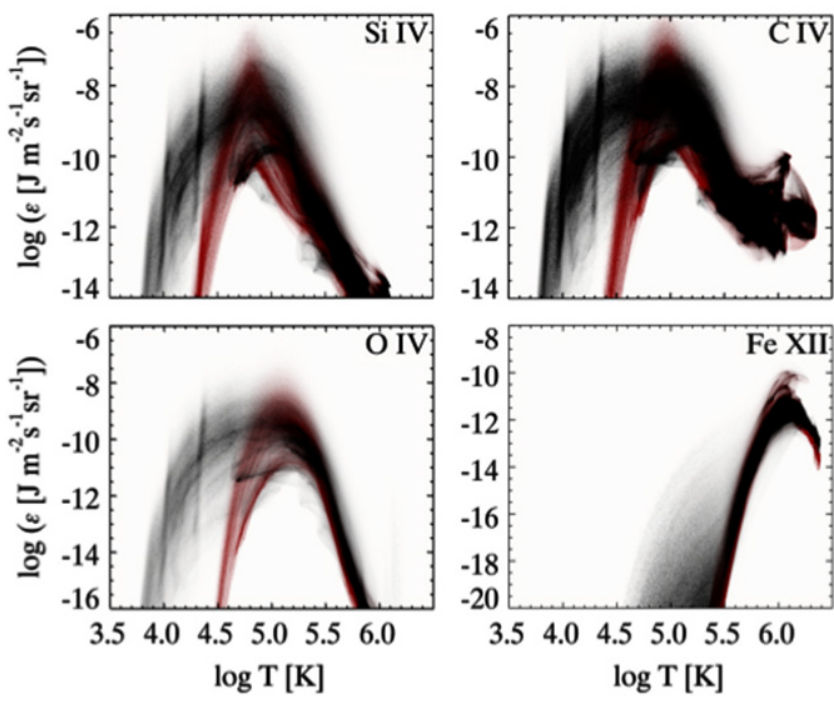

Figure 16. PDF of the logarithmic emissivity as a function of temperature for the ions of interest. The black PDF shade shows the NEQ distributions, while the red shade shows what the distributions would be under SE conditions.

run. The difference in intensity is mainly due to the accumulation of ions at different temperature regions compared to those assumed by SE. The effect is most noticeable for C IV, as can be seen in Figure 15. A large fraction of the $C$ IV ions are located at lower temperatures than they should be according to SE. C IV has a decreasing recombination rate with decreasing temperature, which means that $\mathrm{C}_{\mathrm{IV}}$ located in a plasma that is cooling has a decreasing recombination rate, leaving a large fraction of the carbon as C IV even though the plasma temperature becomes low. As the density increases with decreasing temperature, the high ion fractions of $\mathrm{C}$ IV at temperatures lower than $\log T=5$. produce a stronger intensity for the lines formed by $\mathrm{C}$ IV. The same effect applies to both $\mathrm{O}$ IV and $\mathrm{Si}$ IV, though to a smaller degree. To show the effect directly, the contribution functions for the ions are shown in Figure 16; there is a considerable contribution to the intensity from temperatures much lower than for SE. Again, we can see that the effect is most prominent for C IV and O IV.

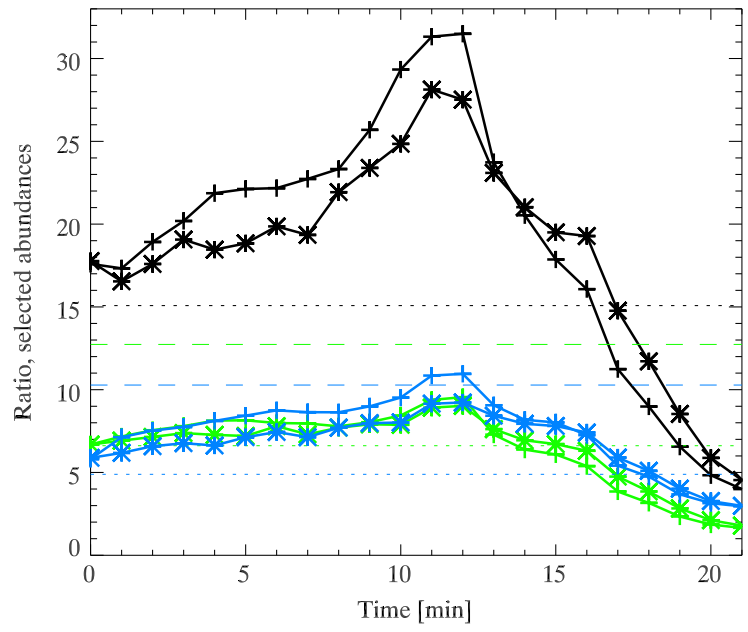

\subsection{NEQ Line Widths}

In the second row of panels in Figure 18 we plot the equivalent nonthermal line widths $\xi$ in units of $\mathrm{km} \mathrm{s}^{-1}$. It should be noted that during the time period for which this analysis is created, the corona is rather cold, so the results for the iron lines are not included, but earlier work (Olluri et al. 2013 b) shows that in a more active and hotter corona, iron will also show NEQ effects.

For $\mathrm{Si}$ IV and $\mathrm{O}$ IV the nonthermal line width is generally at least $2 \mathrm{~km} \mathrm{~s}^{-1}$ larger in the NEQ case, and for C IV it is actually more than $3 \mathrm{~km} \mathrm{~s}^{-1}$ larger. When calculating the nonthermal width in the NEQ case, a thermal width is assumed that is equal to the SE thermal width. As we already have seen in Figure 15, a large fraction of the ions are actually emitted at lower temperatures, so the true thermal width is smaller than the assumed SE thermal width. Consequently, the part of the line broadening from unresolved motions now has to be larger in the NEQ case to attain the same nonthermal line width as in the $\mathrm{SE}$ case. As the nonthermal line width is greater in the NEQ case, that must mean that the larger range of temperatures the emission comes from samples a larger range in velocities, which more than makes up for the lower true thermal width in the NEQ case.

The time evolution of the average nonthermal line widths changes much more rapidly than do the intensities with timescales down to minutes, changing by more than 10 $\mathrm{km} \mathrm{s}^{-1}$ for the apex region for the Si IV line. The largest NEQ effects are seen over the footpoint region for all the lines.

\subsection{NEQ Doppler Shifts}

The time evolution of the Doppler shifts of the synthesized SE and NEQ spectra is presented in the bottom row of panels in Figure 18. The Doppler shifts are generally not affected very much by NEQ, as Doppler shifts depend on the velocities present in the volume where the emissivity is different from zero. So a large shift in Doppler velocities would have to come from a large gradient in vertical velocities with height, and for most of the ions under consideration, the large gradient should be such that the

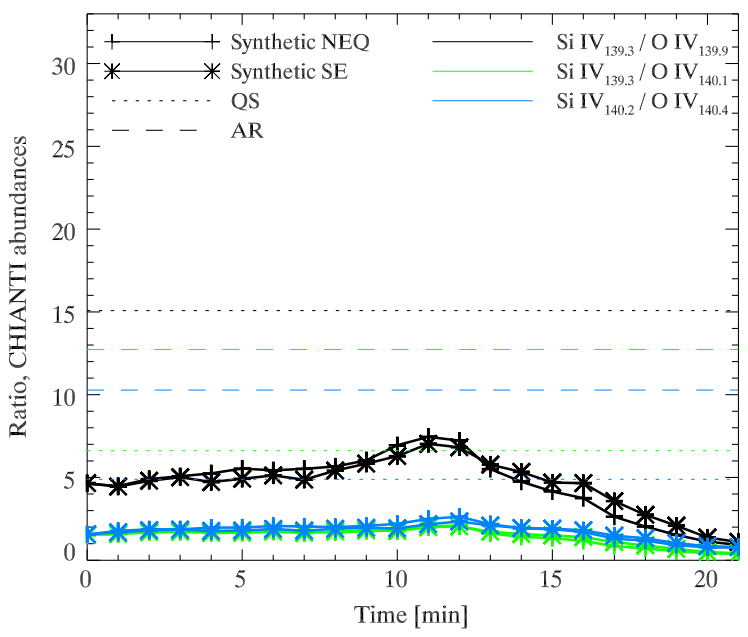

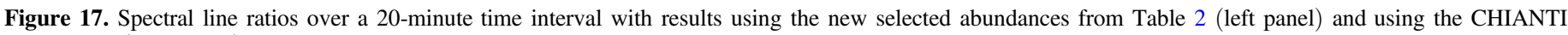

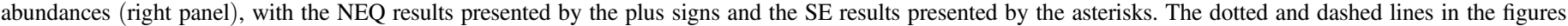
represent the spectral line ratios from QS and AR observations, respectively. 

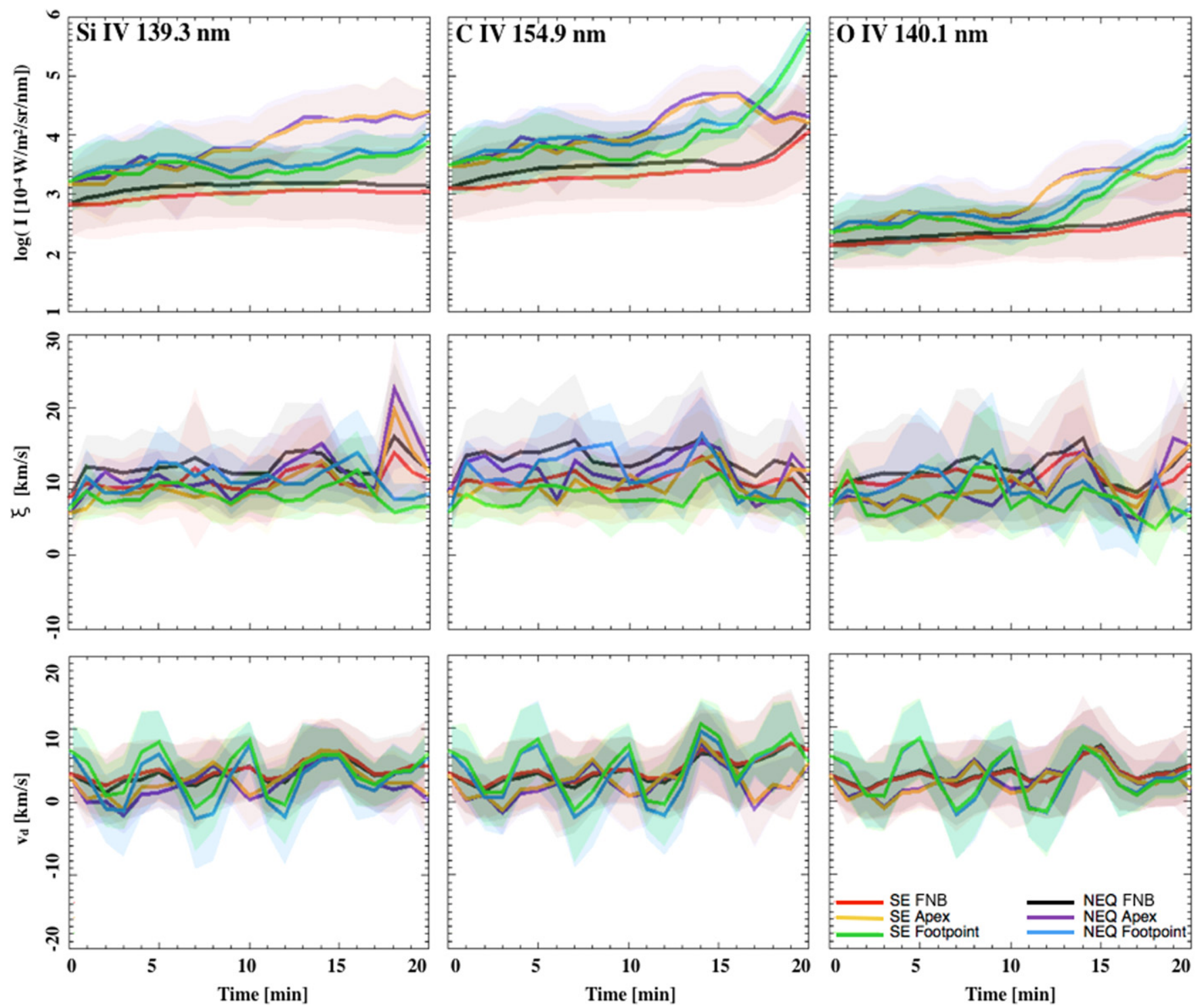

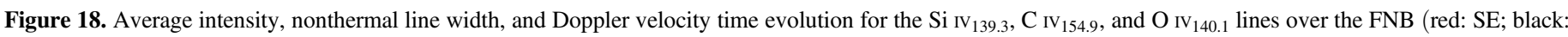
$\mathrm{NEQ}$ ), apex region (yellow: SE; purple: NEQ), and footpoint region (green: SE; blue: NEQ), with the transparent shaded areas representing the $1 \sigma$ standard deviation.

velocities are significantly higher at lower temperatures where the NEQ effects move the ions. The ion most affected is Si IV, but even here the Doppler shifts are at most $3 \mathrm{~km} \mathrm{~s}^{-1}$ smaller for the Si IV lines when NEQ effects are included, seen for the footpoint region 8 minutes into the run.

The Doppler velocities show the 5-minute global oscillation, discussed in Section 2. Nonequilibrium ionization does not seem to have large effects on the average FNB Doppler velocities or the apex Doppler velocities. There is a small effect in the footpoint region, to some extent resulting from the 5minute global wave, where the average Doppler velocities are slightly shifted toward the blue for the Si IV lines and the C IV line, but not for the $\mathrm{O}$ IV lines. This is due to the plasma velocities at the footpoint region, where there are strong upflows advecting the ions upward. Owing to the long ionization times of the ions, they can accumulate at highertemperature regions. This effect is strongest for the Si IV ions, which have the lowest ionization temperature. The effect decreases with increasing ionization temperature and is therefore not visible for the O IV ions.

\subsection{An Instant in Time}

So far we have looked at trends by studying the time evolution of the average spectral properties. It is also instructive to look at how the averages come about. In Figure 19 we show the computational box for the $\mathrm{Si} \mathrm{IV}_{134.9}$ line, both the SE case and the NEQ case, and with log intensity, nonthermal line width, and Doppler shifts. The first noticeable feature is the very prominent intermittency of the images. The line moments show loop-like structures that differ between NEQ and SE on small scales. The smearing to $1^{\prime \prime}$ that has been performed in the analysis so far is quite severe and hides a large amount of this kind of structure. An example of where the smearing would have a large effect is in the apex region, where both large positive and large negative Doppler shifts are present within a very small area; this is also visible in the footpoint region for intensity, Doppler shifts, and nonthermal line widths.

The log intensity, nonthermal line width, and Doppler shift seem to be more accentuated in NEQ than for the SE case. The images show larger excursions from the average in NEQ, and 

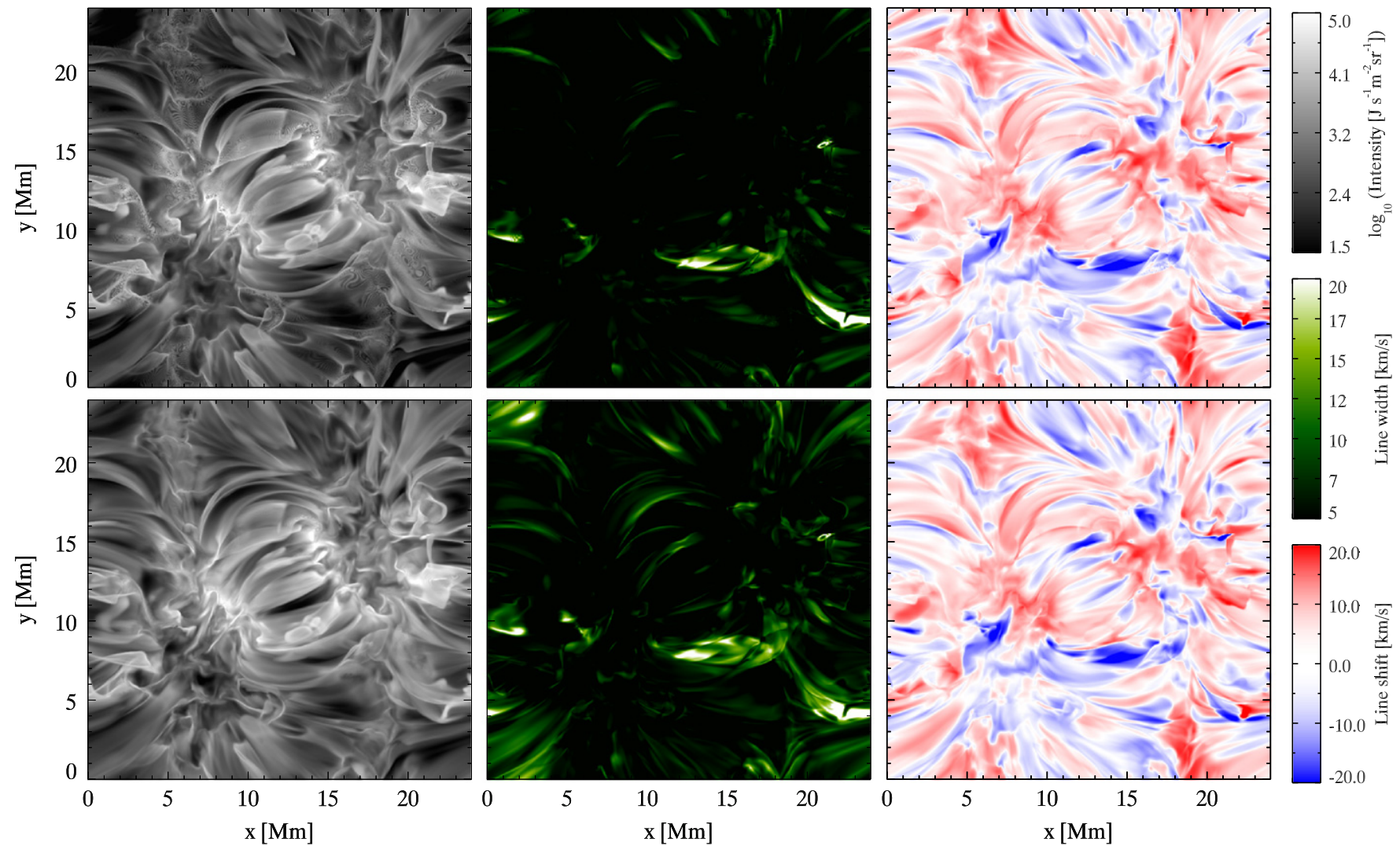

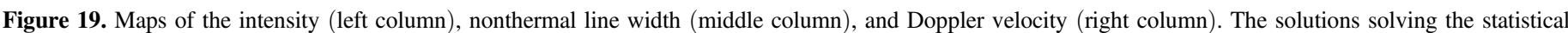

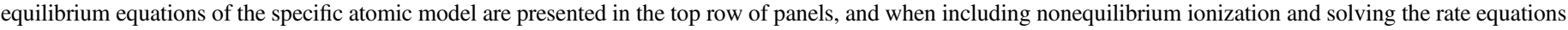
for the atomic model in the bottom row of panels, for the $\mathrm{Si}_{\mathrm{IV}_{139.3}}$ line.

(An animation of this figure showing similar maps for the other lines is available.)

this at very small scales. The intensity is concentrated at the footpoint region and the apex region, while the line widths are very low in the footpoint region. The Doppler shift generally shows a downflow, but there are large patches showing upflows at both footpoints. In addition, the Doppler shifts are generally very intermittent. The intensities in Figure 19 show that there are fewer places where there are very low intensities in the NEQ case. On the other hand, the nonthermal line width shows much more signal in the NEQ case, even in places where there is no signal in SE. The SE and NEQ maps of Doppler shifts show almost no differences, just as the time evolutions of the averages from Figure 18, already discussed in Section 9.5.

To show the differences nonequilibrium ionization introduces in the spectral line properties, we have made scatterplots of the SE and NEQ results of a single snapshot for the $\mathrm{Si} \mathrm{IV}_{139.3}$, $\mathrm{C}_{\mathrm{IV}_{154.9}}$, and $\mathrm{O} \mathrm{IV}_{140.1}$ lines presented from top to bottom in the panels of Figure 20, where the log intensity, nonthermal line width, and Doppler velocity are presented in the panels from left to right. The data presented here are after the native resolution has been rebinned to a pixel size equivalent to $1^{\prime \prime}$, where most of the small-scale features visible in Figure 19 are consequently reduced. Nonetheless, there are still significant departures between the SE results and the NEQ results. The intensities show increasing departures from equilibrium with decreasing intensity for all lines considered. The nonthermal line widths, on the other hand, seem to increase for the Si IV lines and the $\mathrm{C}_{\mathrm{IV}_{154.9}}$ line, while decreasing for the $\mathrm{O}_{\mathrm{IV}}$ lines. The largest NEQ impact is for the $\mathrm{CIV}_{154.9}$ line, where the nonthermal line width can increase up to $30 \mathrm{~km} \mathrm{~s}^{-1}$, where the equivalent $\mathrm{SE}$ result is around $10 \mathrm{~km} \mathrm{~s}^{-1}$. For the $\mathrm{O}_{\mathrm{IV}}$ ions its the opposite, where nonthermal line widths around $10 \mathrm{~km} \mathrm{~s}^{-1}$ in SE are close to $0 \mathrm{~km} \mathrm{~s}^{-1}$ in NEQ. The Doppler velocities are on average very close to equilibrium, represented by the overlapping red and blue lines in the right row of panels in Figure 20, but there is a scatter about the lines, showing the largest spread for the Si IV lines and the $\mathrm{C}_{\mathrm{IV}_{154.9}}$ line.

\section{DISCUSSION}

As part of the IRIS data product, 3D cubes and synthetic diagnostics based on models very similar to those described here are publicly released ${ }^{5}$ in order to aid the interpretation of the IRIS lines. These lines are largely formed in the upper chromosphere and lower TR. The goal of this paper is to see how well the so-called realistic models are able to reproduce solar observables formed in the TR and lower corona. This is not necessary to prove that the models are "correct", but rather to show that they are a tool that can be used to model solar emission, and thereby become a tool for improving our understanding of the phenomena that occur in this region of the atmosphere. Numerical models of the photosphere are already at the state where they can be used for quite detailed comparisons with observations (Beeck et al. 2012; Pereira et al. 2013a). A similar development is now underway for the middle to upper solar chromosphere (Leenaarts et al. 2013b,

http://sdc.uio.no/search/simulations 

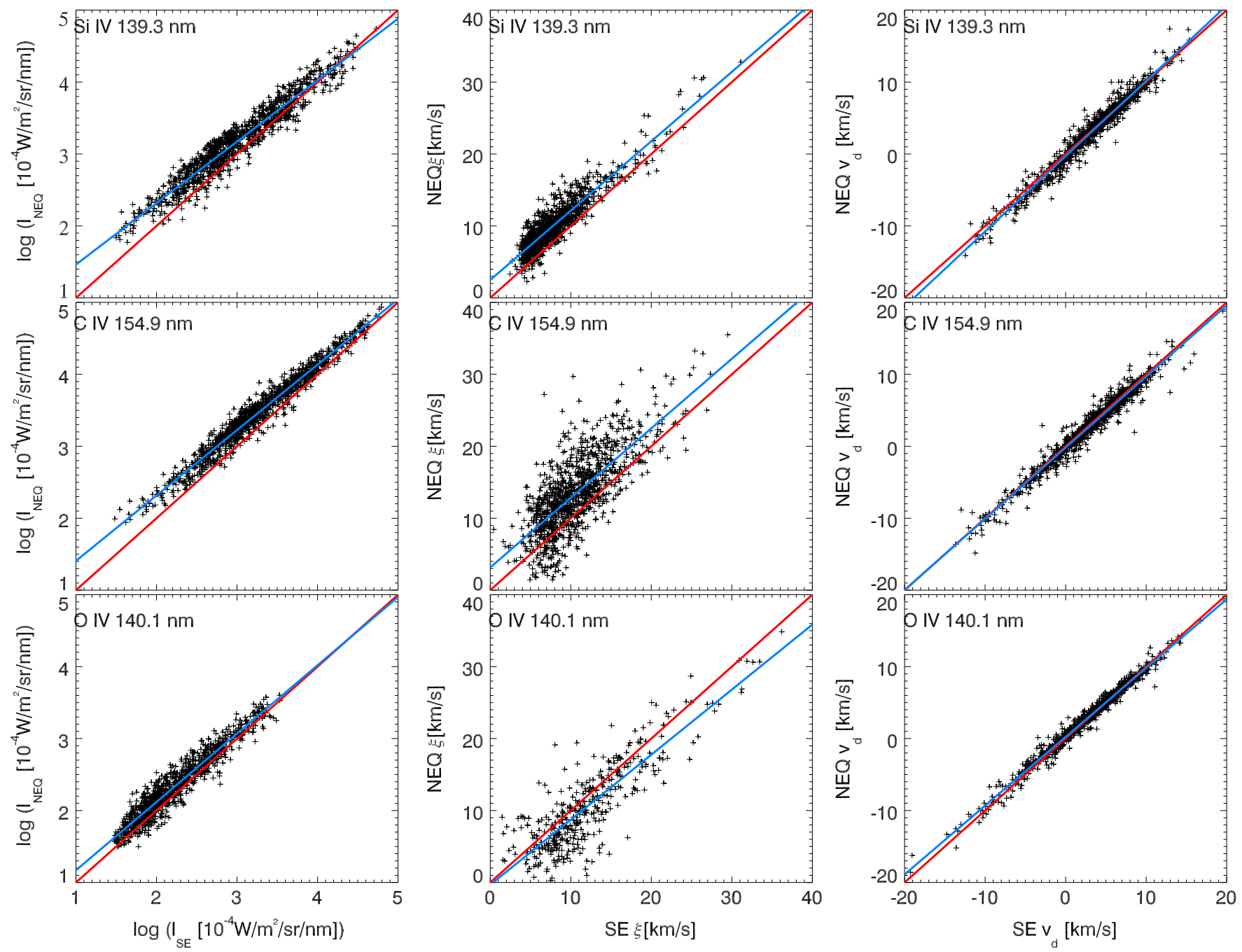

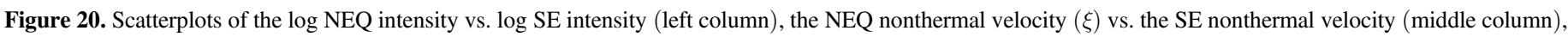

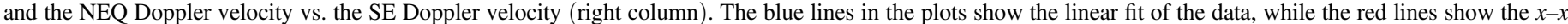
lines, where the difference between these lines shows the average deviation from equilibrium on the spectra.

2013a; Pereira et al. 2013b; M. Carlsson, in preparation), and diagnostics based on initial "realistic" models of the TR (Peter et al. 2006; Hansteen et al. 2010; Guerreiro et al. 2013) show promising similarities with solar observables such as the average Doppler shift.

Previously, with 1D models, there have been two major problems in reconciling observations of TR spectral lines with synthetic diagnostics: it has proven very difficult to reproduce total absolute intensities in the lower TR, and no good explanation of the average redshift measured in lower TR lines has been forthcoming. The advent of 3D models improved this situation markedly; all the published "realistic" 3D models seem able to capture some of these basic properties of the observed TR. The difference between them and the 1D models presumably lies in much better description of the magnetic topology the 3D models offer; most lower TR emission seems indeed to originate in low-lying largely cool loops (Guerreiro et al. 2013), not often captured in 1D models that attempt to encompass the entirety of the corona.

The emerging synthetic intensities of the models described here are able to reproduce the observed absolute intensities to within a factor of two for the most important IRIS lines formed in the TR under the assumption that the silicon abundance is set to its deduced "coronal" value (Feldman 1992), while the oxygen abundance is reduced to $A_{O}=8.69$, i.e., the Asplund et al. (2009) value. Using these values, we find that the siliconto-oxygen intensity ratios lie close to that which is observed, for both the "QS" and "AR" regimes of our numerical experiment. Excepted from this overall "good fit" are the lower TR lines presumed to be optically thick: the $\mathrm{He}_{\text {II }}$ and $\mathrm{C}_{\text {II }}$ lines. Lines from both of these ions show synthetic intensities smaller than what is measured, when calculated assuming that they are optically thin. However, taking radiative transfer into account, B. Rathore \& M. Carlsson (2015, private communication) have calculated examples of the $\mathrm{C}$ II lines that lie closer to their observed values. The He II lines should likewise be treated with full radiative transfer and out of ionization equilibrium before conclusions can be drawn about whether or not the discrepancy is indicative of a model failure. For the iron lines considered, formed at (low) coronal temperatures, the low synthetic intensities are presumably telling us that the modeled heating process is not vigorous enough to produce sufficient material at coronal temperatures. This shows that the model studied here applies to QS, and not so much to AR.

Considered at the native resolution of the numerical simulation, $48 \mathrm{~km}$, synthetic line widths are much smaller than 
what is observed. However, the synthetic nonthermal line widths are sensitive to resolution. When considered at a typical "observed" resolution, say, 1", the synthetic line widths increase by some $20 \%$ and thus lie closer to what is observed, increasing even more when a PSF is applied. That resolution is important is also hinted at by the He $\mathrm{II}_{25.6}$ line, whose synthetic line profile is much greater than the other synthetic line profiles. This line has a contribution function significantly different from zero over a broader range of temperatures than the other lines studied; it therefore necessarily samples a wider range of densities and velocities than the other lines, summing up to a wider profile. Nevertheless, we believe that the discrepancy in Doppler width is real in the sense that structure is found on much smaller scales on the Sun than what is possible to model at the resolution of the presented simulations.

Observations show a large range in average Doppler shifts, but when considering large regions, a net redshift, peaking at $10 \mathrm{~km} \mathrm{~s}^{-1}$ at temperatures of $10^{5} \mathrm{~K}$ while decreasing toward higher and lower TR temperatures, is found. There are indications of a transition to a net blueshift at coronal temperatures. The average synthetic Doppler shifts for the full simulation box follow this trend very nicely. The synthetic Doppler shifts are more or less independent of the resolution chosen to compute them. However, we have not studied the effects of spectral resolution, which might play an important role. The synthesized spectra have a spectral resolution between $10^{-4} \mathrm{~nm}$ and $10^{-5} \mathrm{~nm}$, depending on the line, while measured spectra of the Sun have been done at resolutions between $10^{-3}$ and $10^{-4} \mathrm{~nm}$. It is interesting to note that the average Doppler shifts are quite dependent on the type of region considered: regions dominated by footpoint emission seem to be shifted significantly toward the blue as compared to apex regions or the total emission spanned by the computational domain.

We have carried out correlation studies of various combinations of the intensity, Doppler shift, and nonthermal line width. We find that previously observed results are well reproduced, with the exception of the observed correlation between intensity and nonthermal line width. Observations show strong positive correlations in the temperature range $2 \times 10^{4}-10^{5} \mathrm{~K}$, decreasing toward higher temperatures, while the synthetic spectra produce small negative correlations that increase with increasing temperature of line formation. The results of the synthetic spectra change to match those of observations, though with smaller correlation coefficients, when the native data are convolved with a Gaussian PSF. Correlation studies can thus lead to misinterpretations of atmospheric conditions, owing to photon leakage in the detectors here represented by the PSF.

The nature of the line broadening is complicated since turbulent motion, UFS, and wave motion may each cause broadening of a spectral line (e.g., de Pontieu et al. 2012). The fact that the simulation is able to reproduce more or less correct absolute intensities but struggles with producing broad nonthermal line widths indicates that small-scale dynamics are not well reproduced in these models.

Though the general trends are the same under both SE and nonequilibrium, there are several effects worth noting that occur when nonequilibrium ionization is included in the formation of the emitting ion populations. The results presented here find that all ions considered, except the Fe XII ions, are out of ionization equilibrium. The TR ions of $\mathrm{Si}$ IV, $\mathrm{C}$ IV, and $\mathrm{O}$ IV are all, in general and on average, emitting at lower temperatures than what is assumed in SE. The Fe XII ions are more or less in equilibrium in the model atmosphere we have studied, but earlier studies have shown that for a hotter corona, even these lines will be out of equilibrium (Olluri et al. 2013b). The resulting synthesized spectra, averaged over the FNB, show overall higher intensities for the TR lines than what is found in SE. The increase is modest, ranging between $10 \%$ and $70 \%$ depending on the spectral line, as well as the region. The most affected lines are the $\mathrm{CIV}_{154.9}$, with increases in the FNB, apex, and footpoint regions of $50 \%, 20 \%$, and $70 \%$, respectively, and the $\mathrm{Si} \mathrm{IV}_{139.4}$ with increases of $40 \%, 10 \%$, and $30 \%$, respectively. A sensible estimate is that the intensity increases by roughly $20 \%$ when NEQ effects are taken into account for the TR lines.

The large NEQ effects on the intensity maps, nonthermal line widths, and Doppler shifts are almost lost with large integration times, which, when combined with studying the moments at low resolution, at times can make the spectra appear similar to those found under the assumption of SE. When considering single snapshots at full resolution, the NEQ effect is obvious.

The consequence of the ions being found at lower temperatures than that assumed with SE reduces the thermal line width and will throw off estimates of the nonthermal line widths and therefore the small-scale dynamics of the lower TR. In spite of this, the nonthermal line widths are in general higher when the spatial resolution is set to $1^{\prime \prime}$, for the TR lines investigated when NEQ ionization is considered, with the largest differences seen over the footpoint region. The fact that the ions are also found at much lower temperature means that the ions are also emitting from deeper layers of the atmosphere, and we are consequently sampling larger volumes when integrating along the line of sight, giving higher nonthermal line widths.

Since the Si IV lines are used for temperature diagnostics of the TR, the impact of the ion being out of ionization equilibrium gives an overestimate of the temperature. The same is the case for the $\mathrm{O}$ IV lines, which are used for electron density diagnostics. In Olluri et al. (2013a) the effects of nonequilibrium ionization are discussed, and an overestimate of more than one order of magnitude of the electron density is found.

We have shown that the stellar atmosphere code Bifrost is able to reproduce observables compatible with and comparable to observed solar data of the TR. This study shows that the simulations can be trusted to produce data similar enough to observed solar data to make meaningful and instructive comparisons with the IRIS data. The data provided by IRIS will give insight into the properties of the upper chromosphere and lower TR, as long as one is careful when interpreting the data, remembering that nonequilibrium effects do have an effect on the spectra.

This research was supported by the Research Council of Norway through the grant "Solar Atmospheric Modeling" and ERC Grant agreement No. 291058. Computing time was allocated by the Norwegian metacenter for computational science (NOTUR) and computing project s1061 from the High End Computing (HEC) division of NASA. B.D.P. was supported by NASA grants NNX11AN98G, NNM12AB40P, and NNG09FA40C. We would additionally like to thank the VAPOR team (Clyne \& Rast 2005; Clyne et al. 2007a; 
Clyne et al. 2007b) for making the software available to the scientific community and consequently aiding us with appropriate visualization tools for this paper. And last but not least, we thank Dr. Philip Judge for his help and guidance in understanding nonequilibrium ionization and the atomic processes involved.

\section{REFERENCES}

Akiyama, S., Doschek, G. A., \& Mariska, J. T. 2003, AdSpR, 32, 1137 Antiochos, S. K. 1982, NASA STI/Recon Technical Report No. 83, 29163 Asplund, M., Grevesse, N., Sauval, A. J., \& Scott, P. 2009, ARA\&A, 47, 481 Athay, R. G. 1984, ApJ, 287, 412

Athay, R. G., Gurman, J. B., Henze, W., \& Shine, R. A. 1983, ApJ, 265, 519 Bartoe, J.-D. F., Brueckner, G. E., Cook, J. W., Dere, K. P., \& Morrison, M. D

(ed.) 1986, Results from the NRL instruments on Spacelab 2, 24th AIAA Aerospace Sciences Meeting

Beeck, B., Collet, R., Steffen, M., et al. 2012, A\&A, 539, A121

Bradshaw, S. J., \& Cargill, P. J. 2010, ApJL, 710, L39

Brekke, P., Hassler, D. M., \& Wilhelm, K. 1997, SoPh, 175, 349

Brekke, P., Kjeldseth-Moe, O., Bartoe, J.-D. F., \& Brueckner, G. E. 1991, ApJS, 75, 1337

Brković, A., Peter, H., \& Solanki, S. K. 2003, A\&A, 403, 725

Brown, C. M., Feldman, U., Seely, J. F., Korendyke, C. M., \& Hara, H. 2008, ApJS, 176, 511

Brueckner, G. E., Cook, J. W., \& Dere, K. P. 1986, BAAS, 18, 675

Carlsson, M., \& Leenaarts, J. 2012, A\&A, 539, A39

Chae, J., Schühle, U., \& Lemaire, P. 1998, ApJ, 505, 957

Chitta, L. P., Kariyappa, R., van Ballegooijen, A. A., et al. 2013, ApJ, 768, 32

Clyne, J., Mininni, P., Norton, A., \& Rast, M. 2007a, NJPh, 9, 301

Clyne, J., Mininni, P., Norton, A., \& Rast, M. 2007b, NJPh, 9, 1

Clyne, J., \& Rast, M. 2005, Proc. Visualization and Data Analysis, ed. R. F. Erbacher et al., 284

Culhane, J. L., Harra, L. K., James, A. M., et al. 2007, SoPh, 243, 19

Curdt, W., Brekke, P., Feldman, U., et al. 2001, A\&A, 375, 591

de Pontieu, B., Carlsson, M., Rouppe van der Voort, L. H. M., et al. 2012, ApJL, 752, L12

De Pontieu, B., Title, A. M., Lemen, J. R., et al. 2014, SoPh, 289, 2733

Dere, K. P., \& Mason, H. E. 1993, SoPh, 144, 217

Doschek, G. A. 2006, ApJ, 649, 515

Doschek, G. A., Bohlin, J. D., \& Feldman, U. 1976, ApJL, 205, L177

Doschek, G. A., Warren, H. P., Mariska, J. T., et al. 2008, ApJ, 686, 1362

Doyle, J. G., Madjarska, M. S., Roussev, I., Teriaca, L., \& Giannikakis, J. 2002, A\&A, 396, 255

Feldman, U. 1992, PhyS, 46, 202

Grevesse, N., \& Sauval, A. J. 1998, SSRv, 85, 161

Gudiksen, B. V., Carlsson, M., Hansteen, V. H., et al. 2011, A\&A, 531, A154

Guerreiro, N., Hansteen, V., \& de Pontieu, B. 2013, ApJ, 769, 47
Handy, B. N., Tarbell, T. D., Wolfson, C. J., Korendyke, C. M., \& Vourlidas, A. 1999, SoPh, 190, 351

Hansteen, V. 1993, ApJ, 402, 741

Hansteen, V. H., Betta, R., \& Carlsson, M. 2000, A\&A, 360, 742

Hansteen, V. H., \& Gudiksen, B. 2005, in ESA Special Publication 592, Solar Wind 11/SOHO 16, Connecting Sun and Heliosphere

Hansteen, V. H., Hara, H., de Pontieu, B., \& Carlsson, M. 2010, ApJ, 718,1070

Hansteen, V. H., \& Wikstøl, Ø. 1994, SSRv, 70, 103

Hara, H., Watanabe, T., Harra, L. K., et al. 2008, ApJL, 678, L67

Harrison, R. A., Sawyer, E. C., Carter, M. K., et al. 1995, SoPh, 162, 233

Judge, P. G., \& Meisner, R. W. 1994, in ESA Special Publication 373, Solar Dynamic Phenomena and Solar Wind Consequences, the Third SOHO Workshop (New York: Cambridge University Press) ed. J. J. Hunt, 67

Kim, S. S., Roh, H.-S., Cho, K.-S., \& Shin, J. 2006, A\&A, 456, 747

Klimchuk, J. A., Ofman, L., \& Davila, J. M. 1997, BAAS, 29, 28

Klimchuk, J. A., Tripathi, D., Bradshaw, S. J., \& Mason, H. E. 2012 Astronomical Society Meeting Abstracts 220, 423.02

Laming, J. M. 2004, ApJ, 614, 1063

Leenaarts, J., Pereira, T. M. D., Carlsson, M., Uitenbroek, H., \& De Pontieu, B. 2013a, ApJ, 772, 89

Leenaarts, J., Pereira, T. M. D., Carlsson, M., Uitenbroek, H., \& De Pontieu, B 2013b, ApJ, 772, 90

Mariska, J. T. 1992, The Solar Transition Region (Cambridge: Cambridge Univ. Press)

Mariska, J. T., Feldman, U., \& Doschek, G. A. 1978, ApJ, 226, 698

Nordlund, A. 1982, A\&A, 107, 1

Olluri, K., Gudiksen, B. V., \& Hansteen, V. H. 2013a, ApJ, 767, 43

Olluri, K., Gudiksen, B. V., \& Hansteen, V. H. 2013b, AJ, 145, 72

Pereira, T. M. D., Asplund, M., Collet, R., et al. 2013a, A\&A, 554, A118

Pereira, T. M. D., Leenaarts, J., de Pontieu, B., Carlsson, M., \& Uitenbroek, H. 2013b, ApJ, 778, 143

Peter, H. 1999, in AIP Conf. Ser. 471, ed. S. T. Suess, G. A. Gary, \& S. F. Nerney, American Institute of Physics Conference Series, 471, 281

Peter, H., Gudiksen, B. V., \& Nordlund, A. 2006, ApJ, 638, 1086

Peter, H., \& Judge, P. G. 1999, ApJ, 522, 1148

Pottasch, S. R. 1964, SSRv, 3, 816

Reale, F. 2010, LRSP, 7, 5

Schmelz, J. T., Reames, D. V., von Steiger, R., \& Basu, S. 2012, ApJ, 755, 33 Skartlien, R. 2000, ApJ, 536, 465

Spadaro, D., Lanza, A. F., Lanzafame, A. C., et al. 2003, ApJ, 582, 486

Walsh, R. W., \& Galsgaard, K. 2000, BAAS, 32, 845

Warren, H. P. 2006, AdSpR, 37, 359

Wilhelm, K. 2000, A\&A, 360, 351

Wilhelm, K., Curdt, W., Marsch, E., et al. 1995, SoPh, 162, 189

Wilhelm, K., Marsch, E., Dwivedi, B. N., \& Feldman, U. 2007, SSRv, 133,103

Winebarger, A. R., Schmelz, J. T., Warren, H. P., Saar, S. H., \& Kashyap, V. L. 2011, ApJ, 740, 2

Woodgate, B. E., Brandt, J. C., Kalet, M. W., et al. 1980, SoPh, 65, 73 